\title{
COMPLEX PROJECTIVE STRUCTURES: LYAPUNOV EXPONENT, DEGREE AND HARMONIC MEASURE
}

\author{
BERTRAND DEROIN AND ROMAIN DUJARDIN
}

\begin{abstract}
We study several new invariants associated to a holomorphic projective structure on a Riemann surface of finite analytic type: the Lyapunov exponent of its holonomy which is of probabilistic/dynamical nature and was introduced in our previous work; the degree which measures the asymptotic covering rate of the developing map; and a family of harmonic measures on the Riemann sphere, previously introduced by Hussenot. We show that the degree and the Lyapunov exponent are related by a simple formula and give estimates for the Hausdorff dimension of the harmonic measures in terms of the Lyapunov exponent. In accordance with the famous "Sullivan dictionary", this leads to a description of the space of such projective structures that is reminiscent of that of the space of polynomials in holomorphic dynamics.
\end{abstract}

\section{INTRODUCTION}

Our purpose in this paper is to introduce several new objects associated with a $\mathbb{C P}^{1}$ structure on a Riemann surface $X$ of finite type, and study their relationships. In the non-compact case, we assume that the projective structure is "parabolic at the cusps", in a sense that will be made precise below.

To such a projective structure $\sigma$, we associate:

- a Lyapunov exponent $\chi(\sigma)$, which was constructed in [22];

- a degree $\operatorname{deg}(\sigma)$ which is simply the (normalized) asymptotic covering degree of the developing map $\tilde{X} \rightarrow \mathbb{P}^{1}(\tilde{X}$ the universal cover of $X)$;

- a family of harmonic measures $\left(\nu_{x}\right)_{x \in \tilde{X}}$ on $\mathbb{P}^{1}$, which generalize the traditional harmonic measures on the limit sets of Kleinian groups (throughout the paper, $\mathbb{P}^{1}$ stands for $\left.\mathbb{C P}^{1}\right)$.

We will show that the Lyapunov exponent and the degree are related by a simple formula, and give estimates for the Hausdorff dimension of the harmonic measures in terms of $\chi$. We give several applications of these ideas to the study of the space $P(X)$ of (parabolic) projective structures on $X$, in particular revealing new aspects of the famous Sullivan dictionary between rational and Möbius dynamics on $\mathbb{P}^{1}$.

Before proceeding to a detailed presentation of these results, let us present the main characters of the story.

Date: October 29, 2018.

B.D.'s research was partially supported by ANR-08-JCJC-0130-01, ANR-09-BLAN-0116. 
0.1. Parabolic $\mathbb{P}^{1}$-structures. Let us fix a Riemann surface $X$ of finite type (genus $g$ with $n$ punctures) with negative Euler characteristic eu(X) $=2-2 g-n$, as well as a universal cover $c: \widetilde{X} \rightarrow X$. Throughout this paper, by definition the (unmarked) fundamental group $\pi_{1}(X)$ is the deck group of this covering. Recall that the automorphism group of the Riemann sphere is the group $\operatorname{PSL}(2, \mathbb{C})$ acting on $\mathbb{P}^{1}=\mathbb{C} \cup\{\infty\}$ by the formula $\left(\begin{array}{ll}a & b \\ c & d\end{array}\right) \cdot z=\frac{a z+b}{c z+d}$. By definition a Kleinian group is a discrete subgroup of $\operatorname{PSL}(2, \mathbb{C})$.

It is convenient to define a $\mathbb{P}^{1}$-structure on $X$ in terms of the so-called developmentholonomy pair (dev, hol). Consider a non constant locally injective meromorphic map $\operatorname{dev}: \widetilde{X} \rightarrow \mathbb{P}^{1}$, satisfying the equivariance property $\operatorname{dev} \circ \gamma=\operatorname{hol}(\gamma) \circ \operatorname{dev}$, where hol is a representation $\pi_{1}(X) \rightarrow \operatorname{PSL}(2, \mathbb{C})$. If $A \in \operatorname{PSL}(2, \mathbb{C})$, the pairs $(\operatorname{dev}$, hol $)$ and $(A \circ \operatorname{dev}, A \circ$ holo $A^{-1}$ ) will be declared as equivalent. By definition, a $\mathbb{P}^{1}$-structure is an equivalence class of such pairs. We refer here to the survey paper by Dumas, see [27] for a comprehensive treatment of this notion.

When the surface $X$ is not compact (hence by assumption it is biholomorphic to a compact Riemann surface punctured at a finite set), we restrict ourselves to the subclass of parabolic $\mathbb{P}^{1}$-structures. Such a structure has the following well-defined local model around the punctures: each puncture has a neighborhood which is projectively equivalent to the quotient of the upper half plane by the translation $z \mapsto z+1$.

For instance, the canonical projective structure $\sigma_{\text {Fuchs }}$ induced by uniformization (i.e. viewing $X$ as a quotient of $\mathbb{H}$ under a Fuchsian group) is of this type. More generally, the proof of the Ahlfors finiteness theorem (see [1, Lemma 1]) shows that if $\Gamma$ is any torsion free Kleinian group, and $\Omega$ is the orbit of a discontinuity component, then the induced projective structure on $\Omega / \Gamma$ is parabolic. These have been known as covering projective structures, because the developing map is a covering onto its image in this case [43, 44]. An important example is given by quasi-Fuchsian deformations of the canonical structure $\sigma_{\text {Fuchs }}$.

There are many other examples of parabolic $\mathbb{P}^{1}$-structures. For instance surgery operations such as grafting (see Hejhal's original construction in [37]) may produce a parabolic $\mathbb{P}^{1}$-structure with holonomy a Kleinian group that is not of covering type. Such projective structures are usually called exotic. There are yet other examples of $\mathbb{P}^{1}$-structures: a remarkable theorem of Gallo, Kapovich and Marden [31] asserts that (when $X$ is compact) a representation $\pi_{1}(X) \rightarrow \operatorname{PSL}(2, \mathbb{C})$ is the holonomy of a $\mathbb{P}^{1}$-structure on $X$ (for some Riemann surface structure on $X$ ) if and only if it is non elementary and it lifts to a representation with values in $\mathrm{SL}(2, \mathbb{C})$. In particular, there exist $\mathbb{P}^{1}$-structures with holonomy a non discrete (or even dense) subgroup of $\operatorname{PSL}(2, \mathbb{C})$.

It is also of interest to deal with the case of branched $\mathbb{P}^{1}$-structures, where the local injectivity assumption on the developing map is dropped. Examples include conical metrics of constant curvature equal to 1,0 or -1 , with conical points of angle multiple of $2 \pi$. It turns out that some of the results in the paper carry over to this setting. The necessary adaptations will be explained in Appendix A.

0.2. The degree. Let $\mathbb{H}$ denote the upper half-plane $\{\tau, \Im(\tau)>0\}$, and $g_{P}=\frac{2|d \tau|}{\Im \tau}$ be the Poincaré (or hyperbolic) metric on $\mathbb{H}$, that is the unique complete conformal metric of 
curvature -1 , which is invariant under $\operatorname{Aut}(\mathbb{H}) \simeq \operatorname{PSL}(2, \mathbb{R})$. Taking the pull-back of this metric under any biholomorphism between $\widetilde{X}$ and $\mathbb{H}$ endows $\widetilde{X}$ with a complete conformal metric of curvature -1 invariant under $\pi_{1}(X)$, therefore this metric descends to $X$. It is well known that when $X$ is of finite type, the hyperbolic metric has finite volume.

Recall that a representation $\pi_{1}(X) \rightarrow \operatorname{PSL}(2, \mathbb{C})$ is non elementary if it does not preserve any probability measure on the Riemann sphere. The holonomy of a parabolic projective structure is always non elementary: see [31, Theorem 11.6.1, p. 695] for the compact case, and [16, Lemma 10] for a proof in the case of the fourth punctured sphere, which readily extends to all punctured surfaces.

If $\sigma$ is a parabolic projective structure, we want to define $\delta(\sigma)$ as a nonnegative number counting the average asymptotic covering degree of $\operatorname{dev}_{\sigma}: \widetilde{X} \rightarrow \mathbb{P}^{1}$. For any $x \in \widetilde{X}$ we denote by $B(x, R)$ the ball centered at $x$ of radius $R$ in the Poincaré metric, and by vol the hyperbolic volume.

Definition-Proposition 0.1. Let $X$ be a Riemann surface of finite type and $\sigma$ be a parabolic projective structure on $X$. Choose a developing map $\operatorname{dev}: \widetilde{X} \rightarrow \mathbb{P}^{1}$. Let $\left(x_{n}\right)$ be a sequence of points in $\widetilde{X}$ whose projections $c\left(x_{n}\right)$ stay in a compact subset of $X,\left(R_{n}\right)$ be a sequence of radii tending to infinity, and $\left(z_{n}\right)$ be an arbitrary sequence in $\mathbb{P}^{1}$. Then the limit

$$
\delta=\lim _{n \rightarrow \infty} \frac{\# B\left(x_{n}, R_{n}\right) \cap \operatorname{dev}^{-1}\left(z_{n}\right)}{\operatorname{vol}\left(B\left(x_{n}, R_{n}\right)\right)}
$$

exists, and does not depend on the chosen sequences $\left(x_{n}\right),\left(R_{n}\right)$ nor on the developing map dev. The number $\operatorname{deg}(\sigma)=\operatorname{vol}(X) \delta$ is by definition the degree of the projective structure.

The existence of the limit in (1) is not obvious, in particular due to the possibility of boundary effects. The proof ultimately relies on a result of Bonatti and Gómez-Mont [8] and will be carried out in $\$ 2.1$. Observe that this result is reminiscent from Nevanlinna theory, though the information we obtain is much more precise. We can actually derive the asymptotics of the Nevanlinna theoretic counting function $N(r, \operatorname{dev}, z)$ and characteristic $T(r, \mathrm{dev})$ of the developing map (see [58]) and show that these quantities are governed by the degree. Namely, for every $z \in \mathbb{P}^{1}$ an easy computation shows that

$$
N(r, \operatorname{dev}, z) \underset{r \rightarrow \infty}{\sim} T(r, \operatorname{dev}) \underset{r \rightarrow 1}{\sim} 2 \pi \delta \log \left(\frac{1}{1-r}\right) .
$$

Besides, Nevanlinna theory is known to have connections with Brownian motion, see [14]. In this paper we will explore this relationship from a different point of view.

The reason for introducing the normalized invariant $\operatorname{deg}(\sigma)$ is that $\delta(\sigma)$ is invariant under finite coverings, hence does not behave like a degree.

We also show that projective structures with vanishing degree are exactly the covering projective structures (Proposition 2.3).

0.3. The Lyapunov exponent. The second invariant, the Lyapunov exponent of a parabolic projective structure was defined in our previous work [22]. It depends only on the 
holonomy hol $_{\sigma}$ of the structure, and also on the induced Riemann surface structure on $X$. Fix a basepoint $\star \in X$, in particular an identification between the covering group $\pi_{1}(X)$ and the usual fundamental group $\pi_{1}(X, \star)$. As $X$ is endowed with its Poincaré metric, Brownian motion on $X$ is well-defined. Throughout the paper, Brownian motion will refer to the stochastic process with continuous time whose infinitesimal generator is the hyperbolic laplacian (instead of $\frac{1}{2} \Delta$, which is another usual convention). Let $W_{\star}$ be the Wiener measure on the set of continuous paths $\omega:[0, \infty) \rightarrow X$ starting at $\omega(0)=\star$.

Definition-Proposition 0.2. Let $X$ and $\sigma$ be as above. Define a family of loops as follows: for $t>0$, consider a Brownian path $\varnothing$ issued from $\star$, and concatenate $\left.\varnothing\right|_{[0, t]}$ with a shortest geodesic joining $\varnothing(t)$ and $\star$, thus obtaining a closed loop $\widetilde{\varnothing}_{t}$. Then for $W_{\star}$ a.e. Brownian path $\varnothing$ the limit

$$
\chi(\sigma)=\lim _{t \rightarrow \infty} \frac{1}{t} \log \| \text { hol }\left(\widetilde{\varnothing}_{t}\right) \|
$$

exists and does not depend on $\varnothing$. This number is by definition the Lyapunov exponent of $\sigma$.

Here $\|\cdot\|$ is any matrix norm on $\operatorname{PSL}(2, \mathbb{C})$. The existence of the limit in $(3)$ was established in [22, Def-Prop. 2.1]. As expected it is a consequence of the subadditive ergodic theorem. With notation as in [22], $\chi(\sigma)=\chi_{\text {Brown }}($ hol $)$. Another way to define $\chi(\sigma)$ goes as follows (see [22, Rmk 3.7]: identify $\pi_{1}(X)$ with a Fuchsian group $\Gamma$ and independently random elements $\gamma_{n} \in \Gamma \cap B_{\mathbb{H}}\left(0, R_{n}\right)$, relative to the counting measure. Here $\left(R_{n}\right)$ is a sequence tending to infinity as fast as, say $n^{\alpha}$ for $\alpha>0$. Then almost surely

$$
\frac{1}{d_{\mathbb{H}}\left(0, \gamma_{n}(0)\right)} \log \left\|\operatorname{hol}\left(\gamma_{n}\right)\right\| \underset{n \rightarrow \infty}{\longrightarrow} \chi(\sigma) .
$$

0.4. The harmonic measures. The third object that we associate to a $\mathbb{P}^{1}$-structure on $X$ is a family of harmonic measures $\left\{\nu_{x}\right\}_{x \in \tilde{X}}$ on the Riemann sphere, indexed by $\widetilde{X}$. It can be defined in several ways. The following appealing presentation was introduced by Hussenot in his $\mathrm{PhD}$ thesis [39]:

Definition-Proposition 0.3 (Hussenot). Let $X$ be a Riemann surface of finite type and $\sigma$ be a parabolic projective structure on $X$. Choose a representing pair (dev, hol). Then for every $x \in \tilde{X}$, and $W_{x}$ a.e. Brownian path starting at $\omega(0)=x$, there exists a point $\mathrm{e}(\omega)$ on $\mathbb{P}^{1}$ defined by the property that

$$
\frac{1}{t} \int_{0}^{t} \operatorname{dev}_{*}\left(\delta_{\omega(s)}\right) d s \underset{t \rightarrow+\infty}{\longrightarrow} \delta_{\mathrm{e}(\omega)} .
$$

The distribution of the point $\mathrm{e}(\omega)$ subject to the condition that $\omega(0)=x$ is the measure $\nu_{x}$.

For covering $\mathbb{P}^{1}$-structures, we recognize the classical harmonic measures on the limit set.

Another definition of the harmonic measures is based on the so-called Furstenberg boundary map, which was designed in [30], based on the discretization of Brownian motion in $\mathbb{H}$ 
(see also [53, Theorem 3] for a different approach). Furstenberg shows that if $\Gamma \subset \operatorname{PSL}(2, \mathbb{R})$ is a cofinite Fuchsian group and $\rho: \Gamma \rightarrow \operatorname{PSL}(2, \mathbb{C})$ is a non-elementary representation, there exists a unique measurable equivariant mapping $\theta: \mathbb{P}^{1}(\mathbb{R}) \rightarrow \mathbb{P}^{1}$ defined a.e. with respect to Lebesgue measure (here $\mathbb{P}^{1}(\mathbb{R})$ is viewed as $\partial \mathbb{H}$ ) Choose a biholomorphism $\widetilde{X} \simeq \mathbb{H}$, thereby identifying $\pi_{1}(X)$ with a cofinite Fuchsian group. For $\tau \in \mathbb{H}$, recall that the classical harmonic measure $m_{\tau}$ is a probability measure with smooth density on $\mathbb{P}^{1}(\mathbb{R})$, defined as the exit distribution of Brownian paths issued from $\tau$. The harmonic measure $\nu_{x}$ is then defined by $\nu_{x}=\theta_{*} m_{\tau}$, where $x \in \widetilde{X}$ corresponds to $\tau \in \mathbb{H}$, and $\theta$ is associated to dev. From this perspective it is clear that, the measures $\nu_{x}$ are mutually absolutely continuous and depend harmonically on $x$.

0.5. The main results. The main result in this paper is the following formula, relating the Lyapunov exponent and the degree of a $\mathbb{P}^{1}$-structure.

Theorem A. Let $\sigma$ be a parabolic holomorphic $\mathbb{P}^{1}$ structure on a hyperbolic Riemann surface $X$ of finite type. Let as above $\chi(\sigma), \delta(\sigma)$, and $\operatorname{deg}(\sigma)$ respectively denote the Lyapunov exponent, the unnormalized degree and the degree of $\sigma$. Then the following formula holds:

$$
\chi(\sigma)=\frac{1}{2}+2 \pi \delta(\sigma)=\frac{1}{2}+\frac{\operatorname{deg}(\sigma)}{|\operatorname{eu}(\mathrm{X})|} .
$$

Surprisingly enough, the proof is based on the ergodic theory of holomorphic foliations. Indeed, to any representation $\rho: \pi_{1}(X) \rightarrow \operatorname{PSL}(2, \mathbb{C})$ one classically associates its suspension, a flat $\mathbb{P}^{1}$-bundle $M_{\sigma} \rightarrow X$ whose monodromy is $\rho$. In more concrete terms, it is the quotient of $\widetilde{X} \times \mathbb{P}^{1}$ under the diagonal action of $\pi_{1}(X)$. The horizontal foliation of $\widetilde{X} \times \mathbb{P}^{1}$ descends to a holomorphic foliation on $M_{\sigma}$ transverse to the $\mathbb{P}^{1}$ fibers, with monodromy $\rho$. This "dictionary" between $\mathbb{P}^{1}$-structures and transverse sections of flat $\mathbb{P}^{1}$-bundles, was investigated e.g. in [49].

For $\rho=$ hol $_{\sigma}$, we analyze this foliation from the point of view of Garnett's theory of foliated harmonic measures and currents. This interplay was already explored by Bonatti and Gomez-Mónt [8] and Alvarez [2]. The key of the proof of the theorem is to interpret $\chi$ and $\delta$ as cohomological quantities on $M_{\sigma}$. The idea that foliated Lyapunov exponents can be computed in cohomology stems from the first author's thesis (see [20, Appendice]).

When $X$ is not compact, to prove the result we compactify both $M$ and the foliation. The computations then become much more delicate because the compactified foliation is singular. The details are carried out in Sections 2,3 and 4.

Theorem $\mathrm{A}$ is mostly interesting for the purpose of studying the space of projective structures on $X$. Let $P(X)$ be the space of parabolic projective structures on $X$ which are compatible with the complex structure. It is well known that $P(X)$ is naturally isomorphic to an affine space of quadratic differentials on $X$ of dimension $3 g-3+n$ (see $\$ 6.1$ below for more details). The Bers simultaneous uniformization theorem implies that the Teichmüller space of marked conformal structures on $X$ embeds as a bounded open subset $B(X) \subset$ $P(X)$ (the Bers slice), whose geometry has been extensively studied. 
The Sullivan dictionary is a very fruitful set of analogies between the dynamics of rational transformations on $\mathbb{P}^{1}$ and the theory of Kleinian (and more generally Möbius) groups. In [55], McMullen draws a fundamental parallel between the Bers slice $B(X)$ and the Mandelbrot set. We take one step further here by relating $P(X)$ and the space of polynomials. In this respect, Theorem $\mathrm{A}$ should be understood as the analogue of the familiar ManningPrzytycki formula [51, 61] for the Lyapunov exponent of the maximal entropy measure of a polynomial. This analogy should be used as a guide for the forthcoming results.

It was shown in [22] that $\sigma \mapsto \chi(\sigma)$ is a continuous (Hölder) plurisubharmonic (psh for short) function on $P(X)$, hence it follows from Theorem A that deg is continuous and psh, too. In addition we see that $\chi(\sigma)$ reaches its minimal value $\frac{1}{2}$ exactly when $\operatorname{deg}(\sigma)=0$. As already observed, deg $=0$ on $\overline{B(X)}$, so in particular $\chi=\frac{1}{2}$ there.

A first result which parallels exactly the dynamics of polynomials concerns the Hausdorff dimension of the harmonic measures.

Theorem B. Let $X$ be a hyperbolic Riemann surface of finite type and $\sigma$ be a parabolic projective structure on $X$. Let as above $\chi$ be its Lyapunov exponent and $\left(\nu_{x}\right)_{x \in \tilde{X}}$ be the associated family of harmonic measures. Then for every $x$,

$$
\operatorname{dim}_{H}\left(\nu_{x}\right) \leq \frac{1}{2 \chi} \leq 1
$$

Furthermore $\operatorname{dim}_{H}\left(\nu_{x}\right)=1$ if and only if $\sigma$ belongs to the closure of the Bers slice $B(X)$.

Notice that since the measures $\nu_{x}$ are mutually absolutely continuous, $\operatorname{dim}_{H}\left(\nu_{x}\right)$ is independent of $x$, so abusing notation, we often simply denote it as $\operatorname{dim}_{H}(\nu)$. The proof is an adaptation of Ledrappier [47, Thm 1].

So, as in the polynomial case, Theorem B provides an alternate approach to the classical bound $\operatorname{dim}_{H}(\nu) \leq 1$ for the harmonic measure on boundary of discontinuity components of finitely generated Kleinian groups, which follows from the famous results of Makarov [50] and Jones-Wolff [41]. In addition, with this method we are also able to show that $\operatorname{dim}_{H}(\nu)<1$ when the component is not simply connected. Indeed we have the more precise bound $\operatorname{dim}_{H}(\nu) \leq \frac{A}{2 \chi}$, where $0 \leq A \leq 1$ is an invariant of the flat foliation, and $A<1$ when hol is not injective.

We also see that the value of the dimension of the harmonic measures detects exotic quasifuchsian structures, that is, projective structures with quasifuchsian holonomy which do not belong to the Bers slice.

As a third application of Theorem A, we recover a result due to Shiga [63].

Theorem C. Let $X$ be a hyperbolic Riemann surface of finite type (of genus $g$ with $n$ punctures). The closure of the Bers embedding $B(X)$ is a polynomially convex compact subset of the space $P(X) \simeq \mathbb{C}^{3 g-3+n}$ of holomorphic projective structures on $X$. As a consequence, $B(X)$ is a polynomially convex (or Runge) domain. 
Recall that a compact set $K$ in $\mathbb{C}^{N}$ is polynomially convex if $\widehat{K}=K$, where

$$
\widehat{K}=\left\{z \in \mathbb{C}^{N},|P(z)| \leq \sup _{K}|P| \text { for every polynomial } P\right\} .
$$

An open set $U \subset \mathbb{C}^{N}$ is said to be polynomially convex (or Runge) if for every $K \Subset U$, $\widehat{K} \subset U$. The theorem may be reformulated by saying that $\overline{B(X)}$ is defined by countably many polynomial inequalities of the form $|P| \leq 1$. This is not an intrinsic property of Teichmüller space, but rather a property of its embedding into the space $P(X)$ of holomorphic projective structures on $X$ (as opposed to the Bers-Ehrenpreis theorem that Teichmüller spaces are holomorphically convex).

Shiga's proof is based on the Grunsky inequality on univalent functions. Only the polynomial convexity of $B(X)$ was asserted in [63], but the proof covers the case of $\overline{B(X)}$ as well. Our approach is based on the elementary fact that the minimum locus of a global psh function on $\mathbb{C}^{N}$ is polynomially convex.

In [22] we showed that $T_{\text {bif }}:=d d^{c} \chi$ is a bifurcation current, in the sense that its support is precisely the set of projective structures whose holonomy representation is not locally structurally stable in $P(X)$. Equivalently, the complement $\operatorname{Supp}\left(T_{\mathrm{bif}}\right)^{c}$ is the interior of the set of projective structures with discrete holonomy $P_{D}(X)$. A theorem due to Shiga and Tanigawa [64] and Matsuzaki [54] asserts that $\operatorname{Int}\left(P_{D}(X)\right)=P_{Q F}(X)$, the set of projective structures with quasifuchsian holonomy, so we conclude that $\operatorname{Supp}\left(T_{\text {bif }}\right)=\left(P_{Q F}(X)\right)^{c}$.

Analogous bifurcation currents have been defined for families of rational mappings on $\mathbb{P}^{1}$. It turns out that the exterior powers $T_{\text {bif }}^{k}$ are interesting and rather well understood objects in that context (see [26] for an account). In particular, in the space of polynomials of degree $d$, the maximal exterior power $T_{\text {bif }}^{d-1}$ is a positive measure supported on the boundary of the connectedness locus, which is the right analogue in higher degree of the harmonic measure of the Mandelbrot set [25].

For bifurcation currents associated to spaces of representations, nothing is known in general about the exterior powers $T_{\text {bif }}^{k}$. In our situation, we are able to obtain some information.

Theorem D. Let $X$ be a compact Riemann surface of genus $g \geq 2$. Let $T_{\mathrm{bif}}=d d^{c} \chi$ be the natural bifurcation current on $P(X)$. Then $\partial B(X)$ is contained in $\operatorname{Supp}\left(T_{\mathrm{bif}}^{3 g-3}\right)$.

Notice that $3 g-3$ is the maximum possible exponent. It is likely that the support of $T_{\text {bif }}^{3 g-3}$ is much larger than $\partial B(X)$. The reason for the compactness assumption here is that the proof is based on results of Otal [60] and Hejhal [38] that are known to hold only when $X$ is compact.

If $\gamma$ is a geodesic on $X$, we let $Z(\gamma)$ be the subvariety of $P(X)$ defined by the property that $\operatorname{tr}^{2}(\operatorname{hol}(\gamma))=4$ (i.e. $\operatorname{hol}(\gamma)$ is parabolic or the identity). As a consequence of Theorem $D$ and of the equidistribution theorems of [22] we obtain the following result, which contrasts with the description of $\partial B(X)$ "from the inside" in terms of maximal cusps and ending laminations ([56, 9], see also [46] for a nice account). 
Corollary E. For every $\varepsilon>0$ there exist $3 g-3$ closed geodesics $\gamma_{1}, \ldots, \gamma_{3 g-3}$ on $X$ such that $\partial B(X)$ is contained in the $\varepsilon$-neighborhood of $Z\left(\gamma_{1}\right) \cap \cdots \cap Z\left(\gamma_{3 g-3}\right)$.

We observe that the value 4 for the squared trace is irrelevant here. As the proof will show, the result holds a.s. when $\gamma_{1}, \cdots, \gamma_{k}$ are independent random closed geodesics of length tending to infinity.

\subsection{Notation.}

$\mathbb{P}^{1}=\mathbb{P}^{1}(\mathbb{C})$ is the Riemann sphere. $z$ often denotes the variable in $\mathbb{P}^{1}$

$\mathbb{H}$ the upper half plane. $\tau$ a variable in $\mathbb{H}$

$X$ the finite type Riemann surface on which is defined a projective structure

$\sigma$ a parabolic projective structure on $X$.

$\operatorname{dev}=\operatorname{dev}_{\sigma}: \widetilde{X} \rightarrow \mathbb{P}^{1}$ a developing map

hol $=$ hol $_{\sigma}: \pi_{1}(X) \rightarrow \operatorname{PSL}(2, \mathbb{C})$ the holonomy representation

$\pi: M_{\sigma} \rightarrow X$ the flat $\mathbb{P}^{1}$-bundle over $X$

$\mathbb{P}_{x}^{1}=\pi^{-1}(x)$ the fiber over $x \in X$.

$\varpi: \widetilde{X} \times \mathbb{P}^{1} \rightarrow M_{\sigma}$ the quotient map.

$s: X \rightarrow M_{\sigma}$ a holomorphic section of $\pi$

$\mathcal{F}$ the holomorphic foliation defined by the flat connexion

$T$ the harmonic current on $M_{\sigma}$

$\bar{M}_{\sigma}, \overline{\mathcal{F}}, \bar{s}, \bar{T}$, etc. are the compactifications of the corresponding objects when $X$ is not compact.

\section{HARMONiC MEASURES AND HARMONIC CURRENTS}

In this section we introduce a number of geometric objects which that will be fundamental in our study: the suspension $M_{\sigma}$ (as well as its compactification $\bar{M}_{\sigma}$ ), and the foliation $\mathcal{F}$ (resp. $\overline{\mathcal{F}}$ ). We also study the ergodic theoretic properties of $\mathcal{F}$, by way of three closely related, though slightly different tools: a family of harmonic measures on the fibers of $M_{\sigma}$, a foliated harmonic current, and its associated foliated harmonic measure.

1.1. Generalities. We fix once for all a Riemann surface $X$ of finite type, that is, $X$ is biholomorphic to a compact Riemann surface $\bar{X}$ with finitely many points deleted. We assume that $X$ has negative Euler characteristic. If necessary, we endow $X$ with its hyperbolic metric, which is of finite volume.

A $\mathbb{P}^{1}$-bundle over a Riemann surface $X$ is a holomorphic fibration $M \rightarrow X$ with $\mathbb{P}^{1}$ fibers. It is always the projectivization of a rank 2 holomorphic vector bundle over $X$. If the Riemann surface is compact, the compact complex surface $M$, being a $\mathbb{P}^{1}$-bundle over a curve, is algebraic (by the GAGA principle), thus in particular it is Kähler. We refer to [3, V.4].

A holomorphic $\mathbb{P}^{1}$-bundle always admits a smooth section $X \rightarrow M$ (and even a holomorphic one, see [3, V.4, p. 139]). When $X$ is compact, the parity of the self-intersection of such a smooth section depends only on the fibration; it is even iff $X$ is diffeomorphic to the trivial bundle $X \times \mathbb{P}^{1}$, and odd otherwise. 
Let $s$ and $f$ respectively denote a smooth section and a fiber of $M \rightarrow X$, then we have that

$$
H^{2}(M, \mathbb{C}):=\mathbb{C}[s] \oplus \mathbb{C}[f],
$$

where $[s]$ and $[f]$ are the cohomology classes dual to $s$ and $f$ respectively. (Throughout this paper, we consistently identify the section $s$ and its graph in $M$ ).

In particular, since we can always choose $s$ to be holomorphic, and since $M$ is Kähler, we obtain an isomorphism between the Dolbeaut cohomology group $H_{\bar{\partial}}^{1,1}(M, \mathbb{C})$, the BottChern cohomology group $H_{\partial \bar{\partial}}^{1,1}(M, \mathbb{C})$, and $H^{2}(M, \mathbb{C})$.

1.2. Parabolic flat $\mathbb{P}^{1}$-bundles. Given a parabolic projective structure $\sigma$ on $X$, we introduce the flat $\mathbb{P}^{1}$-bundle $\mathbb{P}^{1} \rightarrow M_{\sigma} \stackrel{\pi}{\rightarrow} X$ with monodromy hol $\sigma$, namely the quotient of the flat bundle $\widetilde{X} \times \mathbb{P}^{1}$ under the action of $\pi_{1}(X)$ given by

$$
\gamma(x, z)=\left(\gamma x, \operatorname{hol}_{\sigma}(\gamma) z\right)
$$

We denote by $\varpi: \widetilde{X} \times \mathbb{P}^{1} \rightarrow M_{\sigma}$ the natural projection. Also, we let $\mathcal{F}$ be the holomorphic foliation on $M_{\sigma}$ obtained by taking the quotient of the horizontal fibration $\widetilde{X} \times\{z\}$ of $\widetilde{X} \times \mathbb{P}^{1}$.

When $X$ is not compact, we can compactify the bundle $M_{\sigma}$ as a bundle over $\bar{X}$. The flat connexion $\nabla$ extends as a meromorphic connexion $\bar{\nabla}$ on $\bar{M}_{\sigma}$, and the foliation $\mathcal{F}$ extends as a singular holomorphic foliation $\overline{\mathcal{F}}$.

Here are the details. Consider the following model for a $\mathbb{P}^{1}$-bundle over the unit disk equipped with a meromorphic flat connexion having a pole over 0 , defined by the differential equation

$$
\frac{d v}{d u}=\frac{i}{2 \pi u}
$$

in coordinates $(u, v) \in \mathbb{D} \times \mathbb{C}$. We denote the induced foliation on $\mathbb{D} \times \mathbb{P}^{1}$ by $\mathcal{F}_{m}$. The monodromy of the connexion around $u=0$ is the parabolic map $v \mapsto v+1$. Since by assumption the holonomy representation is parabolic, we can glue this local model to each of the cusps of $M_{\sigma}$ to obtain the desired $\mathbb{P}^{1}$-bundle $\bar{M}_{\sigma}$ over $\bar{X}$ equipped with a meromorphic flat connexion $\bar{\nabla}$ and singular holomorphic foliation $\overline{\mathcal{F}}$.

1.3. Parabolic $\mathbb{P}^{1}$-structures and holomorphic sections of flat $\mathbb{P}^{1}$-bundles. Let now $\operatorname{dev}: \widetilde{X} \rightarrow \mathbb{P}^{1}$ be a developing map of the parabolic $\mathbb{P}^{1}$-structure $\sigma$. The map $\widetilde{X} \ni x \mapsto$ $(x, \operatorname{dev}(x)) \in \widetilde{X} \times \mathbb{P}^{1}$ is $\pi_{1}(X)$-equivariant, hence it descends to a section $s: X \rightarrow M_{\sigma}$ of the bundle $M_{\sigma} \rightarrow X$. This section will play an important role in what follows.

Lemma 1.1. The section $s$ extends to a section $\bar{s}: \bar{X} \rightarrow \bar{M}_{\sigma}$ which is transverse to the foliation $\overline{\mathcal{F}}$.

Proof. To make the compactification more explicit, consider a neighborhood $N$ of a puncture equipped with a coordinate $x$ in which the projective structure is defined in the punctured unit disk by its developing map $x \mapsto \log x$ (see 80.1 ). Write $x=\exp (2 i \pi \tau)$ with 


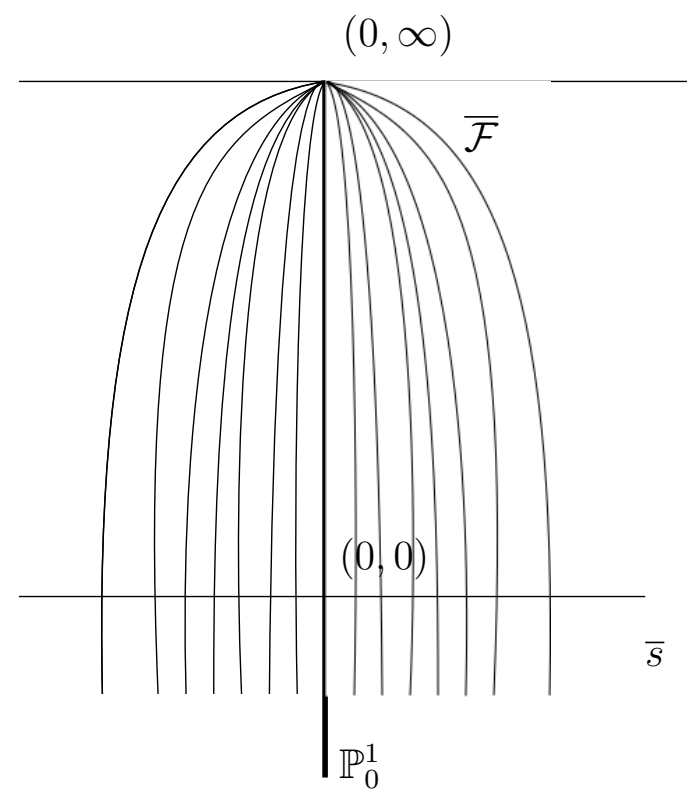

FiguRE 1. Schematic view of the compactified foliation

$\tau \in \mathbb{H}$. The $\mathbb{P}^{1}$-bundle $M_{\sigma}$ over the punctured disk $N \simeq \mathbb{D}^{*}$ is the quotient of $\mathbb{H} \times \mathbb{P}^{1}$ by the cyclic group generated by $(\tau, z) \mapsto(\tau+1, z+1)$. The map

$$
(\tau, z) \longmapsto(u=\exp (2 i \pi \tau), v=z-\tau)
$$

is invariant under this transformation, and it maps the horizontal foliation to the foliation defined by $d z=d v+d \tau=\left(d v+\frac{d u}{2 i \pi u}\right)=0$. Hence (8) provides the identification between the bundle $M_{\sigma}$ over $N \simeq \mathbb{D}^{*}$ and the model (7).

The section $s$ of $M_{\sigma}$ is defined in the coordinates $(\tau, z)$ to be the diagonal $z \mapsto(z, z)$, so in the coordinates $(u, v)$ it is given by $u \mapsto(u, 0)$. Hence the section $s$ extends as a section $\bar{s}$ of $\overline{M_{\sigma}}$.

1.4. Fiberwise harmonic measures. Fix an biholomorphism between $\widetilde{X}$ and $\mathbb{H}$, thereby identifying $\pi_{1}(X)$ with a lattice $\Gamma$ in $\operatorname{PSL}(2, \mathbb{R})$. Recall from [30, 53] that if $\rho: \Gamma \rightarrow$ $\operatorname{PSL}(2, \mathbb{C})$ is a non elementary representation, there exists a unique (Lebesgue) measurable $\rho$-equivariant map $\Phi: \mathbb{P}^{1}(\mathbb{R}) \rightarrow \mathbb{P}^{1}$ defined almost everywhere. Likewise, if $\operatorname{Prob}\left(\mathbb{P}^{1}\right)$ denotes the compact convex set of probability measures on $\mathbb{P}^{1}$ (endowed with the weak* topology), then the map $a \in \mathbb{P}^{1}(\mathbb{R}) \mapsto \delta_{\Phi(a)} \in \operatorname{Prob}\left(\mathbb{P}^{1}\right)$ is the unique measurable $\rho$ equivariant map.

A measurable family of probability measures $\left(m_{\tau}\right)_{\tau \in \mathbb{H}}$ on $\mathbb{P}^{1}$ is said to be harmonic if for every test function $\psi$, the function $\mathbb{H} \ni \tau \mapsto \int \psi m_{\tau}$ is harmonic.

Proposition 1.2. Let $\Gamma$ be a lattice in $\operatorname{PSL}(2, \mathbb{R})$ and $\rho: \Gamma \rightarrow \operatorname{PSL}(2, \mathbb{C})$ be a non elementary representation. Then there exists a unique measurable family of probability measures $\left\{\nu_{\tau}\right\}_{\tau \in \mathbb{H}}$ on $\mathbb{P}^{1}$ such that : 
(i) $\tau \mapsto \nu_{\tau}$ is harmonic;

(ii) $\nu_{\gamma \tau}=\rho(\gamma)_{*} \nu_{\tau}$ for every $\gamma \in \pi_{1}(X)$ and every $\tau \in \mathbb{H}$.

In particular, we have the formula

$$
\text { for every } \tau \in \mathbb{H}, \nu_{\tau}=\frac{1}{\pi} \Phi_{*}\left(\frac{\Im \tau d a}{|\tau-a|^{2}}\right),
$$

and these measures coincide with those defined in Definition 0.3 .

The family $\left\{\nu_{\tau}, \tau \in \mathbb{H}\right\}$ will be simply referred to as the family of harmonic measures associated to the $\mathbb{P}^{1}$-structure.

Proof. It will be convenient to view $\mathbb{P}^{1}(\mathbb{R})$ as the boundary of the upper half plane. First, it follows from the Poisson formula and the equivariance of $\Phi$ that (9) defines a family of harmonic measures $\nu_{\tau}$ satisfying (i) and (ii).

To establish the uniqueness statement, fix a family of probability measures $\left\{\nu_{\tau}^{\prime}\right\}_{\tau \in \mathbb{H}}$ on $\mathbb{P}^{1}$ satisfying (i) and (ii). Then, Fatou's theorem on boundary values on bounded harmonic functions implies that there is a measurable map $\widehat{\nu}^{\prime}: \partial \mathbb{H} \rightarrow \operatorname{Prob}\left(\mathbb{P}^{1}\right)$ with values in the set of probability measures on $\mathbb{P}^{1}$, such that the Poisson formula holds, namely for every $\tau \in \mathbb{H}$

$$
\nu_{\tau}^{\prime}=\frac{1}{\pi} \int_{\mathbb{R}} \frac{\Im \tau}{|\tau-a|^{2}} \widehat{\nu}^{\prime}(a) d a .
$$

The map $\widehat{\nu}^{\prime}$ is $\rho$-equivariant since the family $\left\{\nu_{\tau}^{\prime}\right\}_{\tau \in \mathbb{H}}$ is. Hence by the observations preceding the proposition, we get that a.s. $\widehat{\nu^{\prime}}(a)$ is the Dirac mass at $\Phi(a)$, and we are done.

The fact that these measures coincide with the ones from Definition-Proposition 0.3 follows from this uniqueness. Indeed, the family of measures defined by Definition 0.3 clearly satisfies the equivariance property (ii). To check (i) we adapt the classical argument for the harmonic dependence of the harmonic measures with respect to the starting point. Indeed let $\mathrm{e}(\omega)$ be the endpoint mapping defined in Definition 0.3 . Let $B \subset \mathbb{P}^{1}$ be any Borel set. Let us prove that $u: x \mapsto \mathbb{P}_{x}(e(\omega) \in B)$ is harmonic. For this, identify $\widetilde{X}$ with the unit disk and $D$ be a small disk centered at $x \in \widetilde{X}$. For $\omega \in \Omega_{x}$, let $T=\inf \{t>0, \omega(t) \notin D\}$. It follows from the strong Markov property of Brownian motion in $\widetilde{X}$, that

$$
u(x)=\mathbb{E}_{x}\left(\mathbb{P}_{\omega(T)}(\mathrm{e}(\omega(\cdot-T)) \in B)=\mathbb{E}_{x}(u(\omega(T)))=\int_{\partial D} u .\right.
$$

Therefore $u$ satisfies the mean value property and the result follows.

Remark 1.3. The map $\Phi$ is in general not injective on any full measure subset of $\mathbb{P}^{1}(\mathbb{R})$. However a theorem of Ledrappier shows that this is the case when $\rho$ is faithful and discrete, see [48]. This result will be used in section 5 .

1.5. Harmonic currents. Given a foliated complex surface $(M, \mathcal{F})$ (possibly with singularities), a directed (or foliated) harmonic current (often simply abbreviated as "harmonic current" in the sequel) is a positive current of bidegree $(1,1)$ which is $\partial \bar{\partial}$-closed, and such that $\langle T, \psi\rangle \geq 0$ if $\psi$ is a $(1,1)$ form which is positive along the leaves. 
Such currents have the following local structure outside the $\operatorname{singular} \operatorname{set} \operatorname{sing}(\mathcal{F})$ : in a foliation box biholomorphic to the bidisk $\mathbb{D} \times \mathbb{D}$ in which the foliation is the horizontal fibration, there exists a finite positive measure $m$ on $\mathbb{D}$, and a non-negative bounded measurable function such that

$$
T=\int \varphi[\mathbb{D} \times\{w\}] d m(w)
$$

and moreover $\varphi(., w)$ is harmonic for $m$-a.e. $w \in \mathbb{D}$. Here as usual $[\mathbb{D} \times\{w\}]$ stands for the current of integration on $\mathbb{D} \times\{w\}$. The product $\varphi m$ is a well-defined object, which can be thought of as a transverse measure for the foliation $\mathcal{F}$. In particular, if $C \subset M$ is a holomorphic curve disjoint from $\operatorname{sing}(\mathcal{F})$, we can define the restriction $\left.T\right|_{C}$ of $T$ to $C$, also referred to as the geometric intersection $T \dot{\wedge}[C]$ between $T$ and $C$ ). Observe that the same makes sense for any current of the form (10), whenever harmonic or not.

The existence of a harmonic current directed by the foliation is classical when $M$ is compact and $\mathcal{F}$ is non-singular (see e.g. [34]); the singular case was treated in [7].

Assume now that $\mathcal{F}$ is the foliation by flat sections of $M_{\sigma}$. There is a $1-1$ correspondence between foliated harmonic currents and the fiberwise harmonic measures of $\$ 1.4$. Indeed, consider a foliated harmonic current $T$ on $M_{\sigma}$, normalized so that one (hence all) of its vertical slices is of unit mass. Lifting $T$ to the universal cover $\mathbb{H} \times \mathbb{P}^{1}$, we obtain a harmonic current $\widetilde{T}$ directed by the horizontal fibration, that is invariant with respect to the action of $\Gamma \simeq \pi_{1}(X)$ defined in (6). Restricting to the vertical fibers $\{\tau\} \times \mathbb{P}^{1}$ we get a family of measures $\nu_{\tau}$ which is easily seen to satisfy the assumptions of Proposition 1.2 .

Conversely, any family of measures $\left(\nu_{\tau}\right)_{\tau \in \mathbb{H}}$ on $\{\tau\} \times \mathbb{P}^{1}$ satisfying the assumptions of Proposition 1.2 gives rise to a foliated harmonic current on $M_{\sigma}$. For this, working first on $\mathbb{H} \times \mathbb{P}^{1}$, we construct from (i) a harmonic current $\widetilde{T}$ directed by the horizontal fibration. Indeed, the Poisson formula asserts that $\nu_{\tau}$ is a convex combination of measures of the form $h(\tau, a) \delta_{\Phi(a)}$, where $\tau \mapsto h(\tau, a)$ is harmonic. Then we get $\widetilde{T}$ by taking the corresponding combination of currents of the form $h(\tau, a)[\mathbb{H} \times\{\Phi(a)\}]$. From the equivariance property (ii), $\widetilde{T}$ descends to a foliated harmonic current on $M_{\sigma}$ and we are done.

The following uniqueness statement will be of utmost importance to us. When $X$ is compact it was already established in [24].

Proposition 1.4. Let $X, \sigma$ and $M_{\sigma}$ be as above. The singular foliation $\overline{\mathcal{F}}$ on the compactified suspension $\bar{M}_{\sigma}$ admits a unique normalized foliated harmonic current, carrying no mass on the fibers over the punctures.

Proof. In $M_{\sigma}$, the existence and uniqueness of a foliated harmonic current $T$ giving mass 1 to the vertical fibers follows from the above discussion, together with Proposition 1.2 . Thus, the point is to show that $T$ admits an extension to a harmonic current $\bar{T}$ on $\bar{M}_{\sigma}$ with no mass on the fibers over the punctures, which is then necessarily unique.

Recall that the foliation $\mathcal{F}$ has a well defined rigid model $\mathcal{F}_{m}$ in a neighborhood of each puncture, which was defined in $\$ 1.2$. The key is the following lemma. 
Lemma 1.5. Consider the model foliation $\mathcal{F}_{m}$ on $\mathbb{D} \times \mathbb{P}^{1}$, as defined in $\S 1.2$. Let $T$ be any foliated harmonic current in $\mathbb{D}^{*} \times \mathbb{P}^{1}$, normalized so that the restriction of $T$ to any fiber $u \times \mathbb{P}^{1}, u \neq 0$ is a probability measure. Then the restriction of $T$ to the curve $s^{*}=\left\{0<|u| \leq e^{-2 \pi}, v=0\right\}$ has finite mass.

From this and Lemma 1.1 (see also Figure 1), we deduce that the harmonic current extends to $\mathbb{D} \times \mathbb{P}^{1} \backslash \operatorname{sing}\left(\mathcal{F}_{m}\right)$. It then follows from general extension results for harmonic currents (see e.g. [18, Thm 5]) that it also compactifies at the singular points of $\mathcal{F}_{m}$. The proposition follows.

Proof of Lemma 1.5. The harmonic current $T$ lifts as a harmonic current $\widetilde{T}$ on $\mathbb{H} \times \mathbb{P}^{1}$ which is defined in the $(\tau, z)$-coordinates by a family of measures $\left\{\nu_{\tau}\right\}_{\tau \in \mathbb{H}}$ satisfying

$$
\nu_{\tau+1}=(z+1)_{*} \nu_{\tau},
$$

and depending harmonically on $\tau$. As in the proof of Proposition 1.2, the Poisson formula implies that there exists a family of probability measures $\left\{\nu_{a}\right\}_{a \in \mathbb{R}}$ defined for a.e. $a \in \mathbb{R}$ and depending measurably on $a$, such that for every $\tau \in \mathbb{H}$

$$
\nu_{\tau}=\int_{\mathbb{R}} \frac{\Im \tau}{(\Re \tau-a)^{2}+\Im \tau^{2}} \nu_{a} d a
$$

The equivariance relation (11) implies that

$$
\nu_{a+1}=(z+1)_{*} \nu_{a}
$$

almost everywhere. A canonical example of such a family of measures is given by $\nu_{a}^{\text {can }}=\delta_{a}$ the Dirac mass at the point $a$. It defines a harmonic current $T^{\text {can }}$ (corresponding to the harmonic current on the suspension corresponding to the identity representation).

A fundamental domain for the pull-back of $s^{*}$ in $\mathbb{H} \times \mathbb{P}^{1}$ is the subset $D \times D$ of the diagonal in $\mathbb{H} \times \mathbb{P}^{1}$, where

$$
D=\left\{\frac{-1}{2} \leq \Re \tau \leq \frac{1}{2}, \Im \tau \geq 1\right\} \subset \mathbb{H} .
$$

Therefore, we need to prove that the integral

$$
I=\int_{\mathbb{R}} d a \cdot \int_{D} \frac{\Im \tau}{(\Re \tau-a)^{2}+(\Im \tau)^{2}} \nu_{a}(d \tau)
$$

is finite. Performing the change of variable $a=b+n$ yields

$$
I=\int_{0}^{1} d b \cdot \sum_{n \in \mathbb{Z}} \int_{D} \frac{\Im \tau}{(\Re \tau-(b+n))^{2}+(\Im \tau)^{2}} \nu_{b+n}(d \tau) .
$$

The equivariance relation $(z+n)_{*} \nu_{b}=\nu_{b+n}$ gives

$$
\int_{D} \frac{\Im \tau}{(\Re \tau-(b+n))^{2}+(\Im \tau)^{2}}(z+n)_{*} \nu_{b}(d \tau)=\int_{D-n} \frac{\Im \tau}{(\Re \tau-a)^{2}+(\Im \tau)^{2}} \nu_{a}(d \tau)
$$


where $D-n=\{\tau-n \mid \tau \in D\}$, and we conclude that

$$
I=\int_{0}^{1} d a \cdot \int_{\Im \tau \geq 1} \frac{\Im \tau}{(\Re \tau-a)^{2}+(\Im \tau)^{2}} \nu_{a}(d \tau) \leq 1
$$

since $\nu_{a}$ is a probability measure on $\mathbb{P}^{1}$ and $\frac{\Im \tau}{(\Re \tau-a)^{2}+(\Im \tau)^{2}} \leq 1$ if $\Im \tau \geq 1$. The proof is complete.

Remark 1.6. Identify $\widetilde{X}$ with $\mathbb{H}$ via a biholomorphism. For any $\mathbb{P}^{1}$-structure $\sigma$, the map $\Phi$ can be used to construct a measurable map $M_{\sigma_{\text {Fuchs }}} \rightarrow M_{\sigma}$ mapping biholomorphically every leaf of $\mathcal{F}_{\sigma_{\text {Fuchs }}}$ to a leaf of $\mathcal{F}_{\sigma}$. At the level of the universal covers, this map is simply given by $(\tau, z) \mapsto(\tau, \Phi(z))$. Observe furthermore, that the normalized current $T_{\sigma_{\text {Fuchs }}}$ is mapped to $T_{\sigma}$ (indeed, this holds for the fiber harmonic measures). If in addition the holonomy is faithful with discrete image, Remark 1.3 shows that the foliations $\left(M_{\sigma_{\text {Fuchs }}}, \mathcal{F}_{\sigma_{\text {Fuchs }}}, T_{\sigma_{\text {Fuchs }}}\right)$ and $\left(M_{\sigma}, \mathcal{F}_{\sigma}, T_{\sigma}\right)$ are actually measurably conjugated.

1.6. Foliated harmonic measures: Garnett's theory. In this paragraph we briefly review Garnett's theory of foliated Brownian motion [33] (see also [13]), and adapt it to our non compact situation. Let us define the normalized measure

$$
\mu=\frac{1}{\operatorname{vol}(X)} \operatorname{vol}_{P} \wedge T \text {. }
$$

This measure is a harmonic measure in the sense of Garnett, namely it satisfies the equation $\Delta_{\mathcal{F}} \mu=0$ in the weak sense, here $\Delta_{\mathcal{F}}$ is the leafwise laplacian relative to the leafwise Poincaré metric. (This is immediate from the fact that $T$ itself is harmonic.) We will refer to such measure as foliated harmonic measures.

Let $\Pi=\left\{\Pi_{t}\right\}_{t \geq 0}$ be the Markov semi-group of operators acting on $C_{c}^{0}\left(M_{\sigma}\right)$, whose infinitesimal generator is $\Delta_{\mathcal{F}}$. It is convenient to consider it at the level of the universal cover $\widetilde{X} \times \mathbb{P}^{1}$. There it expresses as

$$
\Pi_{t} f(x, z)=\int_{\widetilde{X}} p(x, y, t) f(y, z) \operatorname{vol}(d y)
$$

where $p(x, y, t)$ is the fundamental solution of the heat equation $\frac{\partial}{\partial t}=\Delta_{\text {Poin }}$ on the hyperbolic plane. Then, since $\mu$ satisfies $\Delta_{\mathcal{F}} \mu=0$, it is invariant under the semi-group $\Pi$.

The following is essentially a reformulation of Proposition 1.4. The proof will be left to the reader.

Proposition 1.7. The measure $\mu$ is the only normalized foliated harmonic measure in the sense of Garnett for $\mathcal{F}$ on $M_{\sigma}$. In particular any measurable subset of $M_{\sigma}$ which is saturated by $\mathcal{F}$ has zero or full $\mu$-measure.

Consider the Markov process on $M_{\sigma}$ induced by the leafwise Brownian motion, with respect to the Poincaré metric (recall that the Brownian motion is generated by the operator $\Delta$ ). More precisely, we let $\Omega^{\mathcal{F}}$ be the set of semi-infinite continuous paths $\omega:[0, \infty) \rightarrow M_{\sigma}$ 
which are contained in a leaf of $\mathcal{F}$, and $\sigma=\left\{\sigma_{t}\right\}_{t \in[0,+\infty)}$ be the shift semi-group acting on $\Omega$ by $\sigma_{t}(\omega)(s)=\omega(t+s)$. Let

$$
W_{\mu}^{\mathcal{F}}:=\int W_{x}^{\mathcal{F}} d \mu(x)
$$

on $\Omega^{\mathcal{F}}$, where $W_{x}^{\mathcal{F}}$ is the Wiener measure on the subset $\Omega_{x}^{\mathcal{F}}$ of paths starting at $x$. We also sometimes use the Wiener space $\left(\Omega^{X}, W^{X}\right)$ of Brownian paths on $X$.

The following proposition is contained in [13, §6]. For the sake of convenience we sketch the proof.

Proposition 1.8. The measure $W_{\mu}^{\mathcal{F}}$ is $\sigma$-invariant and the dynamical system $\left(\Omega^{\mathcal{F}}, \sigma, W_{\mu}^{\mathcal{F}}\right)$ is ergodic.

Proof. Let us first show that $W_{\mu}^{\mathcal{F}}$ is $\sigma$-invariant. Let $E \subset \Omega$ be a measurable subset. By the Markov property, for every $x \in M_{\sigma}$ and every $t \geq 0$ we have that

$$
W_{x}^{\mathcal{F}}\left(\sigma_{t}^{-1} E\right)=\int_{L_{x}} p(x, y, t) W_{y}^{\mathcal{F}}(E) d y .
$$

Consider the function $f:(t, x) \mapsto W_{x}^{\mathcal{F}}\left(\sigma_{t}^{-1} E\right)$. Equation (14) shows that $f$ satisfies the heat equation, with initial condition $f(0, x)=W_{x}^{\mathcal{F}}(E)$, hence for every $t \geq 0, f(t,)=.\Pi_{t} f(0,$.$) .$ Since $\mu$ is invariant under the heat semi-group, we deduce that

$$
W_{\mu}^{\mathcal{F}}\left(\sigma^{-t}(E)\right)=\int_{X_{\rho}} f(t, x) d \mu(x)=\int_{X_{\rho}} f(0, x) d \mu(x)=W_{\mu}^{\mathcal{F}}(E),
$$

hence proving the first part of the proposition.

We now prove that $\left(\Omega^{\mathcal{F}}, \sigma, W_{\mu}^{\mathcal{F}}\right)$ is ergodic. Let $E$ be any $\sigma$-invariant subset. The function $x \mapsto f(0, x)=W_{x}^{\mathcal{F}}(E)$ is then measurable, bounded, and harmonic along $\mu$-a.e. leaf. We claim that it is constant. Indeed, observe that for any $c \in \mathbb{Q}$, the function $g=\max (f, c)$ is leafwise subharmonic on a.e. leaf, so we get that for every $t \geq 0, \Pi_{t} g \geq g$ on a.e. leaf. On the other hand, $\int \Pi_{t} g d \mu=\int g d \mu$, so we infer that on a set of full measure $\Pi_{t} g=g$ holds for every rational $t \geq 0$. This proves that $g$ is harmonic on $\mu$-a.e. leaf. This being true for every $c$, it follows that $f$ is constant along a.e. leaf. Now, since $E$ is shift invariant, belonging to $E$ is a a tail property, so by applying the 0-1 law [13, Prop. 6.5] we infer that $E$ has zero or full measure on a.e. leaf. Applying Proposition 1.7 then concludes the proof.

\section{THE DEGREE}

In this section we introduce the concept of the degree of a $\mathbb{P}^{1}$-structure on $X$. We justify its existence in $\$ 2.1$ by proving Proposition 0.1. Then in $\$ 2.2$, we characterize projective structures with vanishing degree, and in $\$ 2.3$ we show that it can be expressed in terms of cohomological data. 
2.1. Existence of the degree and equidistribution of large leafwise discs. This subsection is devoted to the proof of Definition-Proposition 0.1. Recall that we are given a developing map dev $: \widetilde{X} \rightarrow \mathbb{P}^{1}$ of a parabolic $\mathbb{P}^{1}$-structure with non-elementary holonomy, and wish to show that $\frac{1}{\operatorname{vol}\left(B\left(x_{n}, R_{n}\right)\right)} \#\left\{B\left(x_{n}, R_{n}\right) \cap \operatorname{dev}^{-1}\left(z_{n}\right)\right\}$ converges to some limit $\delta$, independent of the choices. To ease notation we set $\operatorname{vol}\left(R_{n}\right)=\operatorname{vol}\left(B\left(x_{n}, R_{n}\right)\right)$. Using the equivariance we may assume without loss of generality that $\left(x_{n}\right)$ is relatively compact in $\widetilde{X}$. Recall that the graph of the developing map in $\widetilde{X} \times \mathbb{P}^{1}$ descends to a section $s$ of $M_{\sigma}$ transverse to $\mathcal{F}$. Recast in geometric language, we need to show that $\frac{1}{\operatorname{vol}\left(R_{n}\right)} \#\left(B\left(x_{n}, R_{n}\right)\right) \times$ $\left.\left\{z_{n}\right\}\right) \cap \operatorname{Graph}(\mathrm{dev})$ converges to some value $\delta$. Pushing forward by $\varpi$, this amounts to proving that the geometric intersection number

$$
\int_{M_{\sigma}} \varpi_{*}\left(\frac{1}{\operatorname{vol}\left(R_{n}\right)}\left[B\left(x_{n}, R_{n}\right) \times\left\{z_{n}\right\}\right]\right) \dot{\wedge}[s]
$$

converges to $\delta$. Put $\Delta\left(R_{n}\right)=\varpi_{*}\left(\frac{1}{\operatorname{vol}\left(R_{n}\right)}\left[B\left(x_{n}, R_{n}\right) \times\left\{z_{n}\right\}\right]\right)$, which is a current with boundary supported in a leaf of $\mathcal{F}$. It is perhaps useful to stress here that $\Delta\left(R_{n}\right)$, may be decomposed into pieces of varying multiplicities (according to the self- overlapping properties of $\varpi\left(B\left(x_{n}, R_{n}\right)\right)$ ), and that these multiplicities are taken into account in the geometric wedge product $\dot{\wedge}$.

The key is the following equidistribution result for large leafwise discs in parabolic flat $\mathbb{P}^{1}$-bundles, which is due to Bonatti and Gómez-Mont [8], given the positivity of the foliated Lyapunov exponent, a fact that was established in this generality in our previous work [22].

Proposition 2.1. Let $\rho: \pi_{1}(X) \rightarrow \operatorname{PSL}(2, \mathbb{C})$ be a non elementary representation. Let $\left(x_{n}\right)_{n \geq 0}$ be a sequence in $\widetilde{X}$ such that $\left(c\left(x_{n}\right)\right)_{n \rightarrow \infty}$ is relatively compact in $X$. Let $\left(R_{n}\right)$ be a sequence of positive real numbers tending to $+\infty$, and $\left(z_{n}\right)$ be any sequence of points on the Riemann sphere. Then the projection in $M_{\sigma}$ of the sequence of integration currents $\Delta\left(R_{n}\right)=\varpi_{*}\left(\frac{1}{\operatorname{vol}\left(R_{n}\right)}\left[B\left(x_{n}, R_{n}\right) \times\left\{z_{n}\right\}\right]\right)$ converges to $\frac{1}{\operatorname{vol}(X)} T$ when $n$ tends to infinity.

Proof. Let vol denote the Poincaré volume form along the leaves of $\mathcal{F}$. Remark that since all currents are directed by the foliation, the convergence $\Delta\left(R_{n}\right) \underset{n \rightarrow \infty}{\longrightarrow} \frac{1}{\operatorname{vol}(X)} T$ is equivalent to that of $\varpi_{*}\left(\left.\frac{1}{\operatorname{vol}\left(R_{n}\right)} \operatorname{vol}\right|_{B\left(x_{n}, R_{n}\right) \times\left\{z_{n}\right\}}\right)$ towards the measure $\mu:=\frac{1}{\operatorname{vol}(X)} T \wedge \operatorname{vol}$.

By [8, Thm 2], for this it is enough to show that the top Lyapunov exponent of the cocycle induced by $\rho$ over the geodesic flow on $T^{1} X$ is positive. The representation $\rho$ being non elementary, this positivity was shown in [22, Rmk 2.19]. The result follows.

We see that to prove the desired result, it is enough to show that

$$
\int_{M_{\sigma}} \Delta\left(R_{n}\right) \dot{\wedge}[s] \underset{n \rightarrow \infty}{\longrightarrow} \frac{1}{\operatorname{vol}(X)} \int_{M_{\sigma}} T \dot{\wedge}[s] .
$$

We note that it follows from the previous proposition that if $\alpha$ is any smooth form along the leaves of $\mathcal{F},\left\langle\Delta\left(R_{n}\right), \alpha\right\rangle$ converges to $\left\langle\frac{1}{\operatorname{vol}(X)} T, \alpha\right\rangle$. The proof of 115 will be carried 
out in several steps. As it is common in such counting issues, special attention must be paid to boundary effects.

Step 1. Here we prove (15) on compact subsets of $M_{\sigma}$. Since $s$ is a section of $M_{\sigma} \rightarrow X$, it is enough to test the convergence on test functions of the form $\pi^{*} \psi$, with $\psi \in \mathcal{C}_{c}(X)$, which we simply denote by $\psi$, that is, we need to show that $\left\langle\Delta\left(R_{n}\right) \dot{\wedge}[s], \psi\right\rangle \rightarrow \frac{1}{\operatorname{vol}(X)}\left\langle\left. T\right|_{s}, \psi\right\rangle$. Fix $\varepsilon>0$. To lighten notation, we put $B_{n}\left(R_{n}\right)=B\left(x_{n}, R_{n}\right) \times\left\{z_{n}\right\}$.

We first construct a regularization of $\psi[s]$. Since $s$ is transverse to $\mathcal{F}$ we can extend $\psi$ locally around $s$ to be constant along the leaves. Fix a non-negative smooth function $\theta_{\varepsilon}$ : $[0, \infty) \rightarrow[0, \infty)$ with support contained in $[0, \varepsilon]$, and such that $\int_{\mathbb{D}} \theta_{\varepsilon}\left(d_{\text {Poin }}(0, x)\right) \operatorname{vol}(d x)=$ 1. Let now $\Delta$ be a foliated current, expressed as $\Delta=\int \varphi[\mathbb{D} \times\{w\}] d m(w)$ in a flow box around a point of $s$, in which $s$ corresponds to $\{0\} \times \mathbb{D}$. Define a form along the leaves by

$$
(\psi[s])_{\varepsilon}=\theta_{\varepsilon}\left(d_{\mathcal{F}}(\cdot, s)\right) \psi \operatorname{vol}_{\mathcal{F}}
$$

where $d_{\mathcal{F}}\left(\right.$ resp. $\left.\quad \operatorname{vol}_{\mathcal{F}}\right)$ is the leafwise Poincaré distance (resp. volume form). If $\varphi$ is continuous, then clearly $\Delta \wedge(\psi[s])_{\varepsilon}$ is close to $\Delta \dot{\wedge}(\psi[S])$ (when $\Delta=\Delta\left(R_{n}\right)$, this will happen when $\partial \Delta\left(R_{n}\right)$ is far from $\left.s\right)$.

We then write

$$
\begin{aligned}
& \int \Delta\left(R_{n}\right) \dot{\wedge} \psi[s]-\frac{1}{\operatorname{vol}(X)} \int T \dot{\wedge} \psi[s]=\left(\int \Delta\left(R_{n}\right) \wedge(\psi[s])_{\varepsilon}-\frac{1}{\operatorname{vol}(X)} T \wedge(\psi[s])_{\varepsilon}\right)+ \\
& +\int \Delta\left(R_{n}\right) \dot{\wedge} \psi[s]-\Delta\left(R_{n}\right) \wedge(\psi[s])_{\varepsilon}+\frac{1}{\operatorname{vol}(X)} \int T \wedge(\psi[s])_{\varepsilon}-T \dot{\wedge} \psi[s]
\end{aligned}
$$

as a sum of three terms $I+I I+I I I$. Since $(\psi[s])_{\varepsilon}$ is smooth along the leaves, Proposition 2.1 implies that $I$ converges to zero as $n \rightarrow \infty$. Since in the representation $(10)$ the density $\varphi$ of $T$ along the leaves is harmonic, the mean value formula implies that the integral III vanishes.

We will decompose the integral $I I$ as a sum of two contributions. We declare that a point in $B_{n}\left(R_{n}\right) \cap \varpi^{-1}(s)$ is a good intersection if the ball $B_{P o i n}(p, \varepsilon)$ of radius $\varepsilon$ relative to the Poincaré metric is disjoint from $\partial\left(B_{n}\left(R_{n}\right)\right)$. Therefore, $B_{n}\left(R_{n}\right) \cap \varpi^{-1} \operatorname{Supp}(\psi[s])_{\varepsilon}$ is a union of good and bad components. Notice that bad components are contained in a leafwise $2 \varepsilon$-neighborhood of $\partial B_{n}\left(R_{n}\right)$. Pushing forward again by $\varpi$ we let $\Delta_{n}^{\text {bad }}$ be the part of $\Delta\left(R_{n}\right)$ corresponding to bad components and $\Delta_{n}^{\text {good }}$ be its complement (notice that $\Delta_{n}^{\text {good }}$ is larger than the union of good components). Since $\psi$ is constant along the leaves near $s$, by definition of $(\psi[s])_{\varepsilon}$ we get that

$$
\left.\int \Delta_{n}^{\text {good }} \dot{\wedge}(\psi[s])=\int \Delta_{n}^{\text {good }} \wedge(\psi[s])_{\varepsilon}\right) .
$$

To estimate the contribution of the bad part, observe that bad components of $\Delta\left(R_{n}\right)$ become good in $\varpi\left(B_{n}\left(R_{n}+2 \varepsilon\right)\right)$ as well as in the annulus $\varpi\left(B_{n}\left(R_{n}+2 \varepsilon\right) \backslash B_{n}\left(R_{n}-2 \varepsilon\right)\right)$. 
So we infer that

$$
\begin{aligned}
\int \Delta_{n}^{\mathrm{bad}} \dot{\wedge} \psi[s] & \leq \int \frac{1}{\operatorname{vol}\left(R_{n}\right)}\left(\varpi_{*}\left[B_{n}\left(R_{n}+2 \varepsilon\right) \backslash B_{n}\left(R_{n}-2 \varepsilon\right)\right]\right)^{\mathrm{good}} \dot{\wedge} \psi[s] \\
& =\int \frac{1}{\operatorname{vol}\left(R_{n}\right)}\left(\varpi_{*}\left[B_{n}\left(R_{n}+2 \varepsilon\right) \backslash B_{n}\left(R_{n}-2 \varepsilon\right)\right]\right)^{\text {good }} \wedge(\psi[s])_{\varepsilon} \\
& \leq \int \frac{1}{\operatorname{vol}\left(R_{n}\right)} \varpi_{*}\left[B_{n}\left(R_{n}+2 \varepsilon\right) \backslash B_{n}\left(R_{n}-2 \varepsilon\right)\right] \wedge(\psi[s])_{\varepsilon} \\
& \longrightarrow{ }_{n \rightarrow \infty}\left(e^{2 \varepsilon}-e^{-2 \varepsilon}\right) \int T \wedge(\psi[s])_{\varepsilon}=\left(e^{2 \varepsilon}-e^{-2 \varepsilon}\right) \int T \dot{\wedge} \psi[s]=O(\varepsilon)
\end{aligned}
$$

where the convergence in the last line follows from Proposition 2.1 and the fact that $\operatorname{vol}\left(R_{n}+2 \varepsilon\right) \underset{n \rightarrow \infty}{\sim} e^{2 \varepsilon} \operatorname{vol}\left(R_{n}\right)$. We thus conclude that the difference of integrals in $(16)$ is arbitrary small as $n \rightarrow \infty$, and Step 1 is complete.

Step 2. To show that the convergence (15) holds throughout $M_{\sigma}$, we work in the compactification $\bar{M}_{\sigma}$. Let $\mathbb{P}_{p}^{1}$ be the fiber of $\bar{M}_{\sigma} \rightarrow \bar{X}$ over a puncture $p$. We know from Lemma 1.5 that the measure $\bar{T} \dot{\wedge}[\bar{s}]$ has finite mass. Since $\bar{T}$ carries no mass on $\mathbb{P}_{p}^{1}$, from the local picture of $\mathcal{F}$ and $s$ given in $\$ 1.2$, we infer that the measure $\bar{T} \dot{\wedge}[\bar{s}]$ has no atom at $\bar{s}(p)$. Therefore, to prove the desired convergence it is enough to show that the mass of $\Delta\left(R_{n}\right) \dot{\wedge}[s]$ near $\bar{s}(p)$ is uniformly small with $n$.

We use the local model for $\sigma$ near $p$. Fix a coordinate $z$ in which $N(p)$ is identified to $\mathbb{D}^{*}$ and the projective structure is given by $\log z$. Let $N_{\varepsilon}(p)=\{0<|z|<\varepsilon\}$. Then any connected component of $c^{-1}\left(N_{\varepsilon}(p)\right)$ is the interior of a horocycle in $\mathbb{H}$. The crucial point is that the developing map is injective in any component of $c^{-1}\left(N_{\varepsilon}(p)\right)$. In particular the cardinality of $\operatorname{dev}^{-1}\left(z_{n}\right) \cap B_{n}\left(R_{n}\right) \cap c^{-1}\left(N_{\varepsilon}(p)\right)$ is bounded by the number of connected components of $c^{-1}\left(N_{\varepsilon}(p)\right)$ intersecting $B_{n}\left(R_{n}\right)$. Now we observe that there is a universal constant $\alpha>0$ such that if $U$ is such a component, then the area of $B_{n}\left(R_{n}+1\right) \cap U$ is at least $\alpha$. From this we infer that

$$
\#\left\{\operatorname{dev}^{-1}\left(z_{n}\right) \cap B_{n}\left(R_{n}\right) \cap c^{-1}\left(N_{\varepsilon}(p)\right)\right\} \leq \frac{1}{\alpha} \operatorname{vol}_{\mathbb{H}}\left(B_{n}\left(R_{n}+1\right) \cap c^{-1}\left(N_{\varepsilon}(p)\right)\right) .
$$

It is well known that the image of $B_{n}\left(R_{n}+1\right)$ under $c$ becomes asymptotically equidistributed in $X$ as $n \rightarrow \infty$. This may be obtained as a consequence of Proposition 2.1, but it already follows from Margulis [52]. From this and (17), we conclude that

$$
\frac{1}{\operatorname{vol}\left(R_{n}\right)} \#\left\{D^{-1}\left(z_{n}\right) \cap B_{n}\left(R_{n}\right) \cap c^{-1}\left(N_{\varepsilon}(p)\right)\right\}
$$

is bounded by $C \operatorname{vol}_{X}\left(N_{\varepsilon}(p)\right)$ and the result follows.

Remark 2.2. This proof shows that rather than a simple number, it is more precise to view the degree as a positive measure on $X$, defined by the formula $\operatorname{deg}(\sigma)=\pi_{*}(T \dot{\wedge}[s])$. In particular it makes sense to speak of the degree of dev restricted to some $\pi_{1}(X)$-invariant subset of $\tilde{X}$. This measure is canonically associated to the $\mathbb{P}^{1}$-structure on $X$ (that is it does not depend on the chosen developing map). 
The support of the degree measure can be described as follows. Let (dev, hol) be a development-holonomy pair for the structure. Let $\Lambda \subset \mathbb{P}^{1}$ be the limit set of hol. The closed subset $\operatorname{dev}^{-1}(\Lambda)$ being invariant by $\pi_{1}(X)$, it defines a closed subset $\Lambda_{\sigma} \subset X$. This set is canonically associated to the projective structure and does not depend on the chosen development-holonomy pair. An instructive example is given by $\mathbb{P}^{1}$-structures with Fuchsian holonomy. In this case the set $\Lambda_{\sigma}$ is a union of the boundaries of disjoint annuli embedded in $X$. It was studied e.g. by Goldman to prove that such structures are obtained from $2 \pi$-graftings, see 35 .

We claim that the support of the degree is the set $\Lambda_{\sigma}$. Indeed, at the level of the universal cover, the pull-back of the degree is the intersection of $\widetilde{T}$ with the graph of dev. In particular ence by the Harnack inequality, it is absolutely continuous with respect to the pull-back by dev of any harmonic measure, with density bounded from above and below by positive constants. The claim then follows from the fact that the support of the harmonic measures is the limit set of hol. This argument shows more: namely, that the degree has the same Hausdorff dimension of that of the harmonic measures. In particular, our Theorem $B$ shows that the Hausdorff dimension of the degree measure is always smaller than 1 (since the equality case happens only when $\operatorname{deg}(\sigma)=0)$.

Theorem $\mathrm{A}$ shows that the mass of the degree defines a psh function on the moduli space of $\mathbb{P}^{1}$-structures on $X$. It would be interesting to know if the degree is also psh considered as a measure.

2.2. Projective structures with vanishing degree. Recall that $\sigma$ is a covering projective structure on $X$ if its developing map is a covering of some proper open subset $\Omega \subset \mathbb{P}^{1}$. In other words, if $\sigma$ is the quotient of the orbit of a component of the discontinuity set of a Kleinian group. Such projective structures were studied e.g. by Kra [43, 44] who showed that a parabolic projective structure is of covering type if and only if its developing map is not surjective.

Projective structures of covering type may also be characterized in terms of their degree.

Proposition 2.3. Let $X$ be a Riemann surface of finite type and $\sigma$ be a parabolic projective structure on $X$. Then $\operatorname{deg}(\sigma)=0$ if and only if $\sigma$ is of covering type.

Proof. The proof of Definition-Proposition 0.1 shows that the degree vanishes if and only if the support of the foliated harmonic current $T$ is disjoint from $s$. Equivalently, the image of the developing map is disjoint from the support of the harmonic measures. Hence the developing map is not surjective and the result follows from the remarks preceding the proposition.

2.3. Cohomological expression of the degree. Observe that the harmonic current $\bar{T}$ on $\bar{M}_{\sigma}$ naturally defines an element of the dual of the Bott-Chern cohomology group $H_{\partial \bar{\partial}}^{1,1}\left(\bar{M}_{\sigma}, \mathbb{C}\right)$. By the $\partial \bar{\partial}$-lemma, the natural map $H_{\partial \bar{\partial}}^{1,1}\left(\bar{M}_{\sigma}, \mathbb{C}\right) \rightarrow H_{\bar{\partial}}^{1,1}\left(\bar{M}_{\sigma}, \mathbb{C}\right)$ with values in the Dolbeaut cohomology group is an isomorphism. Thus by duality, the current $\bar{T}$ defines a cohomology class $[\bar{T}]$ in $H^{1,1}\left(\bar{M}_{\sigma}, \mathbb{C}\right)$. In more concrete terms, if $\alpha_{1}$ and $\alpha_{2}$ are smooth $(1,1)$ forms defining the same class $[\alpha]$ in $H^{1,1}\left(\bar{M}_{\sigma}, \mathbb{C}\right)$, then $\alpha_{1}-\alpha_{2}=d d^{c} u$ for 
some smooth function $u$, and we get that $\left\langle\bar{T}, \alpha_{1}\right\rangle=\left\langle\bar{T}, \alpha_{2}\right\rangle$. So it makes sense to speak about the pairing between $\bar{T}$ and $\alpha$ which we simply denote it by $\bar{T} \cdot \alpha$. Observe also that any curve $C \subset M_{\sigma}$ admits a class in $H^{1,1}\left(M_{\sigma}\right)$, which is the cohomology class dual to the cycle $C$ (or equivalently, that of the integration current $[\bar{s}]$ ).

Recall from $\$ 2.1$ that $\operatorname{deg}(\sigma)$ is the mass of $\bar{T} \dot{\wedge} \bar{s}$. The next result -presumably part of the folklore- asserts that this geometric intersection number can be computed in cohomology.

Proposition 2.4. Let $\sigma$ be a parabolic projective structure on a Riemann surface of finite type, and $\operatorname{deg}(\sigma)$ be be its degree, as defined in Definition 0.1. Then

$$
\operatorname{deg}(\sigma)=\bar{T} \cdot \bar{s}
$$

Proof. The difficulty is that we cannot simply regularize the integration current $[s]$ within smooth positive forms because, as we will see later, $\bar{s}^{2}<0$. Pick a smooth closed $(1,1)$ form cohomologous to $[\bar{s}]$, and write $[\bar{s}]=\alpha+d d^{c} u$, where $u$ is a quasi-psh function, smooth outside $\bar{s}$, with logarithmic singularities along $s$. Then by definition, $\bar{T} \cdot \bar{s}=\langle T, \alpha\rangle$. Recall that $s$ stays far from the singularities of the foliation $\overline{\mathcal{F}}$ and is everywhere transverse to it. Consider a tubular neighborhood $N_{\varepsilon}$ of $\bar{s}$, such that if $p \in \bar{s}$ and $L_{p}$ is the leaf through $p$, then $L_{p} \cap N_{\varepsilon}$ is a small disk about $p$, contained in a flow box. We modify $u$ by replacing it inside $N_{\varepsilon}$ by any smooth function $u_{\varepsilon}$ such that $u=u_{\varepsilon}$ near $\partial N_{\varepsilon}$. We denote by $u_{\varepsilon}$ the resulting function on $\bar{M}_{\sigma}$. By construction, $[\bar{s}]_{\varepsilon}:=\alpha+d d^{c} u_{\varepsilon}$ is a smooth form cohomologous to $[\bar{s}]$, so $\bar{T} \cdot[\bar{s}]=\left\langle\bar{T},[\bar{s}]_{\varepsilon}\right\rangle$.

Now consider a flow box $\mathbb{B}$ endowed with local coordinates $(z, w) \in \mathbb{D}^{2}$ where $\overline{\mathcal{F}}$ becomes the horizontal foliation and $\bar{s}$ is a vertical graph. Then in this flow box, $[\bar{s}]=d d^{c} v$ for some psh function $v$ and $[\bar{s}]_{\varepsilon}=d d^{c} v_{\varepsilon}$ with $v=v_{\varepsilon}$ in a neighborhood of $\partial \mathbb{D} \times \mathbb{D}$. With notation as in $(10)$, we see that the local contribution of $\left\langle\bar{T},[\bar{s}]_{\varepsilon}\right\rangle$ is equal to

$$
\left.\left\langle\bar{T},[\bar{s}]_{\varepsilon}\right\rangle\right|_{\mathbb{B}}=\int\left(\int_{\mathbb{D} \times\{w\}} \varphi d d^{c} v_{\varepsilon}\right) d m(w)=\int\left(\int_{\mathbb{D} \times\{w\}} \varphi d d^{c} v\right) d m(w)=\left.T \dot{\wedge} \bar{s}\right|_{\mathbb{B}},
$$

where the middle equality follows from the Green formula and the harmonicity of $\varphi$. The result follows.

\section{The LyApunov EXPONENT}

In this section we relate the exponent $\chi$ defined in Definition 0.2 to a foliated Lyapunov exponent introduced by the first author in [20, Appendice]. This leads in $\$ 3.2$ to a cohomological formula for $\chi$ analogous to that obtained for the degree.

3.1. The foliated Lyapunov exponent. Using [22, Proposition 2.2], we start by introducing a Lipschitz family of spherical metrics on $M_{\sigma}$, simpy denoted by $\|\cdot\|$. By this, we mean a smooth family of conformal metrics of curvature +1 on the fibers, with the property that there exists $C>0$ such that for every smooth path $\omega:[0,1] \rightarrow X$, $\log \left\|D h_{\rho}(\omega)\right\|_{\infty} \leq C$ length $(\omega)$, where $h_{\rho}(\omega)$ is the holonomy of $\omega$ and $\left\|D h_{\rho}(\omega)\right\|_{\infty}$ is the supremum of the norm of the fiber derivative relative to the spherical metrics on $\pi^{-1}(\omega(0))$ and $\pi^{-1}(\omega(1))$. We will recall some details of the construction below in 3.2. More generally, 
the notation length $(\omega)$ will stand for the homotopic length of $\omega$, that is, the minimal length of a smooth path homotopic to $\omega$ with fixed endpoints. In particular this notion makes perfect sense for a Brownian sample path.

Since $\mathcal{F}$ is transverse to the fibers, this induces a smooth metric on the normal bundle $N_{\mathcal{F}}$. Later on we will study the extension properties of $\|\cdot\|$ to a singular metric on the fibers of $\bar{M}_{\sigma}$.

Notice that in our situation, the data of a Brownian sample path along a leaf is equivalent to that of its projection on $X$, together with its starting point in the initial fiber. So if the starting point $x$ is given, the projection $\pi$ gives an identification between $W_{x}^{\mathcal{F}}$ and $W_{\pi(x)}^{X}$. In this way we can speak of the holonomy, or homotopic length of a leafwise Brownian path, by simply projecting it to $X$.

We now consider the family of functions

$$
\Omega^{\mathcal{F}} \ni \omega \longmapsto K_{t}(\omega)=\log \left\|D_{\omega(0)} h\left(\left.\omega\right|_{[0, t]}\right)\right\| .
$$

This is a cocycle, in the sense that $K_{t+s}(\omega)=K_{s}(\omega)+K_{t}\left(\sigma_{s} \omega\right)$ for every $t, s \geq 0$. As explained above, the estimate

$$
K_{t}(\omega) \leq C \cdot \operatorname{length}\left(\left.\omega\right|_{[0, t]}\right),
$$

holds, for some $C$ is independent of $\omega$. The superexponential decay of the heat kernel on the hyperbolic plane [17, §5.7] then implies that $K_{t}$ is $W_{\mu}^{\mathcal{F}}$-integrable for every $t \geq 0$. The ergodic theorem shows that for $W_{\mu}^{\mathcal{F}}$-almost every path $\omega$ the $\operatorname{limit} \lambda=\lim _{t \rightarrow \infty} \frac{K_{t}(\omega)}{t}$ exists and does not depend on $\omega$. By definition $\lambda$ is the foliated Lyapunov exponent.

We can now compare $\lambda$ and $\chi$.

Proposition 3.1. Let $\sigma$ be a parabolic projective structure on a Riemann surface of finite type. Let $\chi(\sigma)=\chi_{\mathrm{Brown}}\left(\mathrm{hol}_{\sigma}\right)$ be the Lyapunov exponent of $\sigma$, as defined in $\S 0.3$. Then if $\lambda$ is as above we have $\lambda=-2 \chi(\sigma)$.

The proof relies on the following result:

Lemma 3.2. Assume that $\rho$ is non elementary. Then for every $x \in X$ and $W_{x}^{X}$-a.e. $\omega:[0, \infty) \rightarrow X$ starting at $x$, there exists $r(\omega) \in \mathbb{P}_{x}^{1}$ such that the pointwise convergence

$$
\lim _{t \rightarrow \infty} \frac{1}{t} \log \left\|D_{y} h\left(\left.\omega\right|_{[0, t]}\right)\right\| \rightarrow-2 \chi_{\operatorname{Brown}}(\rho)
$$

holds uniformly on compact subsets of $\mathbb{P}_{x}^{1} \backslash\{r(\omega)\}$. Moreover, the distribution of the exceptional point $r(\omega)$ is the harmonic measure $\nu_{x}$ on $\mathbb{P}_{x}^{1}$.

Proof. In order to apply the Oseledets theorem, consider a measurable trivialization $M_{\sigma} \simeq$ $X \times \mathbb{P}^{1}$ and set $\chi=\chi_{\text {Brown }}(\rho)$. For every continuous $\omega$, and every $t>0$, the map $h_{t}=h_{\left.\omega\right|_{[0, t]}}: \mathbb{P}_{\omega(0)}^{1} \rightarrow \mathbb{P}_{\omega(t)}^{1}$ can be lifted to a matrix $\widetilde{h_{t}}$ in $\operatorname{SL}(2, \mathbb{C})$ which is well defined up to sign. The family $\widetilde{h}=\left\{\widetilde{h_{t}}\right\}_{t \geq 0}$ on $\Omega$ is a cocyle modulo signs, namely it satisfies $\widetilde{h_{t+s}}(\omega)= \pm \widetilde{h_{t}}\left(\sigma_{s}(\omega)\right) \widetilde{h_{s}}(\omega)$ for every $\omega \in \Omega$ and every $s, t \geq 0$. Moreover, from [22, 
Proposition 2.5], we have that for every $x \in X$ and $W_{x}^{X}$ a.e. $\omega$,

$$
\lim _{t \rightarrow \infty} \frac{1}{t} \log \left\|\widetilde{h}_{t}\right\|=\chi
$$

where $\|\cdot\|$ is the matrix norm to the usual hermitian norm $\|\cdot\|_{2}$ on $\mathbb{C}^{2}$. Since $\rho$ is non elementary, by [22, Thm 2.7], $\chi>0$. Since in addition $h$ takes values in $\operatorname{SL}(2, \mathbb{C})$, the Lyapunov exponents of $h$ over $\left(\Omega_{X}, \sigma_{X}, W^{X}\right)$ are $\chi$ and $-\chi$. The Oseledets theorem tells us that for $W$-a.e. $\omega:[0, \infty) \rightarrow X$, there exists a complex line $E=E(\omega) \subset \mathbb{C}^{2}$ such that for every $Y \in \mathbb{C}^{2}, Y \neq 0, \frac{1}{t} \log \left\|\widetilde{h}_{t}(Y)\right\|_{2}$ converge to $-\chi$ as $t \rightarrow \infty$ when $Y \in E$, while this quantity converges uniformly to $\chi$ on compact subsets of $\mathbb{C}^{2} \backslash E$. Finally, we observe that for the usual spherical derivative, we have that

$$
\left\|D h_{t}(y)\right\|_{s}=\frac{\|Y\|_{2}^{2}}{\left\|\widetilde{h}_{t}(Y)\right\|_{2}^{2}} \text {, where } Y \text { is a lift of } y,
$$

hence 18 holds, with $r(\omega)=\mathbb{P} E(\omega) \in \mathbb{P}_{\omega(0)}^{1}$.

It remains to show that the distribution of $r$ when $\omega$ is conditioned to start at $x$ is the harmonic measure $\nu_{x}$. For this, we consider the mapping $\omega^{\mathcal{F}} \ni \omega \mapsto r(\omega)$, which is defined $W_{\mu}^{\mathcal{F}}$-a.e. The push-forward of $W_{\mu}^{\mathcal{F}}$ is a shift invariant measure on $M_{\sigma}$, so we conclude by the unique ergodicity of $\mathcal{F}$ (Proposition 1.7).

Proof of Proposition 3.1. Let $x \in M_{\sigma}$. As observed before, we can identify $\left(\Omega_{\pi(x)}^{X}, W_{\pi(x)}^{X}\right)$ in $X$ and $\left(\Omega_{x}^{\mathcal{F}}, W_{x}^{\mathcal{F}}\right)$ in $M_{\sigma}$ by lifting. Since the harmonic measure $\nu_{\pi(x)}$ on $\mathbb{P}_{\pi(x)}^{1}$ has no atoms, we infer that for $W_{x}^{\mathcal{F}}$ a.e. $\omega$, the point $r(\omega)$ defined in Lemma 3.2 is distinct from $x$. Hence $\lim _{t \rightarrow \infty} \frac{1}{t} \log \left\|D_{x} h\left(\left.\omega\right|_{[0, t]}\right)\right\| \rightarrow-2 \chi$ for $W_{x}$-a.e. $\omega$, and the conclusion follows.

3.2. Cohomological expression of $\chi$. Let $P$ be the set of punctures of $X$. To avoid confusion with the Lyapunov exponent, we denote by eu $(X)$ the Euler characteristic of $X$, $\mathrm{eu}(X)=2-2 g-\# P$. Recall the Gauss-Bonnet formula $\operatorname{vol}(X)=2 \pi|\mathrm{eu}(X)|$.

In this section, we begin the proof of the following result, which will be complete only after proving Theorem A

Proposition 3.3. Let $\sigma$ be a parabolic projective structure on a Riemann surface of finite type with puncture set $P$. Then $\chi(\sigma)=\frac{1}{2|\mathrm{eu}(X)|}\left(N_{\overline{\mathcal{F}}} \cdot \bar{T}+\# P\right)$.

When $X$ is compact $(P=\emptyset)$ this result follows from the cohomological formula derived in [20, Appendice A] for the foliated Lyapunov exponent, and from Proposition 3.1. The proof in the non compact case follows the same strategy but serious technical difficulties arise from the parabolic cusps.

Recall that if $X$ is a complex surface, $E \rightarrow X$ is a holomorphic line bundle, and $\|\cdot\|$ is a hermitian metric on $E$, its curvature form is defined by $\Theta(\|\cdot\|)=\frac{1}{2 i \pi} \partial \bar{\partial} \log \|s\|^{2}$, where $s$ is any non vanishing local holomorphic section of $E$. In our situation we choose a Lipschitz family of spherical metrics on the fibers of $M_{\sigma}$, which, since $\mathcal{F}$ is transverse to the fibers, induces a hermitian metric on the normal bundle $N_{\mathcal{F}}$. Recall that the value of 
the Lyapunov exponent does not depend on this choice. We denote by $\Theta$ the curvature form of this metric.

The first result is obtained exactly as in the compact case [20, Appendice A] (see also [13, $\S 8])$.

Lemma 3.4. $\chi(\sigma)=\frac{\pi}{\operatorname{vol}(X)} \int \Theta \wedge T$.

Proof. We keep notation as in 80.3 . From the fact that $K$ is a cocycle, we deduce that the function $t \mapsto \int K_{t}(\omega) W_{\mu}^{\mathcal{F}}(d \omega)$ is linear. So its slope is equal to its derivative at 0 , and we get that

$$
\lambda=\left.\frac{d}{d t}\right|_{t=0} \int K_{t}(\omega) W_{\mu}^{\mathcal{F}}(d \omega)=\left.\int_{M_{\sigma}} \frac{d}{d t}\right|_{t=0} \mathbb{E}^{x}\left(K_{t}(\omega)\right) d \mu(x) .
$$

Let $x_{0}$ be a point of $X$. We use local coordinates $x=(\xi, \eta)$, to parametrize points in $M_{\sigma}$ via $s(\xi, \eta)$, that is, $x$ belongs to the fiber of $\xi, \eta$ belongs to a neighborhood of $\eta_{0}$ in $\mathbb{P}_{\xi}^{1}$. and $s(\xi, \eta)=h_{\xi, \eta}(x)$ is the flat section passing through the point $x$, defined over a neighborhood of $\xi_{0}$ in $X$. Using the heat equation, the formula (20) can be written in these coordinates

$$
\lambda=\int_{X_{\rho}} \Delta_{\xi} \log \left\|\frac{\partial}{\partial \eta} h_{\xi, \eta}(x)\right\| \mu(d x)
$$

Observe that the curvature form $\Theta$ of the Lipschitz metric on $N_{\mathcal{F}}$, restricted to the tangent bundle of $\mathcal{F}$, is given by the expression

$$
\left.\Theta\right|_{T \mathcal{F}}=\frac{1}{2 i \pi} \partial \bar{\partial}_{\mathcal{F}} \log \left\|\frac{\partial}{\partial \eta} h_{\xi, \eta}(x)\right\|^{2} .
$$

Because we have $\Delta_{\text {Poin }} f \cdot \operatorname{vol}_{\text {Poin }}=2 i \partial \bar{\partial} f$ for every function $f$ defined on the hyperbolic plane, we infer that

$$
\Delta_{\xi} \log \left\|\frac{\partial}{\partial \eta} h_{\xi, \eta}(x)\right\| \operatorname{vol}_{\text {Poin }}=-\left.2 \pi \Theta\right|_{T \mathcal{F}} .
$$

Using the fact that $T \wedge \operatorname{vol}_{\text {Poin }}=\operatorname{vol}(\mathrm{X}) \mu$, we finally obtain

$$
\lambda=-\frac{2 \pi}{\operatorname{vol}(X)} \int \Theta \wedge T,
$$

which, together with Proposition 3.1 finishes the proof of Lemma 3.4 .

When $X$ is compact, it immediately follows from Lemma 3.4 that

$$
\chi=\frac{\pi}{\operatorname{vol}(X)} T \cdot N_{\mathcal{F}}=\frac{1}{2|\mathrm{eu}(X)|} N_{\mathcal{F}} \cdot T,
$$

and the proof of Proposition 3.3 is complete.

In the general case, however, this calculation is no longer valid, and in the remaining part of the argument we need to understand the contribution of the punctures to this formula. For the moment, we content ourselves with the following weakening of Proposition 3.3 . 
Proposition 3.5. Under the assumptions of Proposition 3.3, there exists a universal constant I such that $\chi(\sigma)=\frac{1}{2|\mathrm{eu}(X)|}\left(N_{\overline{\mathcal{F}}} \cdot \bar{T}+I \cdot \# P\right)$.

The proof occupies the remainder of this section. It will be carried out in several steps, mostly dealing with the local study of the model foliation $\mathcal{F}_{m}$ introduced in $\$ 1.2$.

Step 1. A smooth metric.

Let $p \in P$ be a puncture of $X$, and let us work in a neighborhood $\pi^{-1}(U(p))$ of $\pi^{-1}(p)$ in $\bar{M}_{\sigma}$, in the coordinates $(u, v)$ introduced in $\$ 1.2$. We claim that the metric

$$
\|\cdot\|_{s}=|u| \frac{|d v|}{1+|v|^{2}}
$$

defines a smooth metric on $N_{\overline{\mathcal{F}}}$. To see this, observe that a non-vanishing holomorphic section of the normal bundle of $\overline{\mathcal{F}}=\mathcal{F}_{m}$ on $\mathbb{D} \times \mathbb{C}$ in the $(u, v)$-coordinates is defined by $n=\frac{1}{u} \frac{\partial}{\partial v}$. Indeed, $\omega=d u+2 i \pi u d v$ is a form defining $\overline{\mathcal{F}}$, and $\omega(n)=2 i \pi$. We see that $\|n\|_{s}=\frac{1}{1+|v|^{2}}$, so $\|\cdot\|_{s}$ extends smoothly along the line $\{0\} \times \mathbb{C}$. To analyse what happens close to the point $(0, \infty)$, we introduce the new coordinates $(u, V)=\left(u, \frac{1}{v}\right)$. In these coordinates, the foliation is defined by the equation $2 \pi u d V+i V^{2} d u=0$. A non-vanishing section of the normal bundle is then given by $n=\frac{1}{u} \frac{\partial}{\partial V}$, and a straighforward computation yields $\|\cdot\|_{s}=|u| \frac{|d V|}{1+|V|^{2}}$. Hence the situation is symmetric and we conclude that $\|\cdot\|_{s}$ defines a smooth metric on $N_{\overline{\mathcal{F}}}$, as claimed.

Step 2. The Lipschitz metric.

Here we give an explicit expression for a Lipschitz family of spherical metrics on $M_{\sigma}$ close to $p$. Recall that a model for the bundle $\pi^{-1}(\mathcal{U}(p)) \subset M_{\sigma}$ is the quotient of $\mathbb{H} \times \mathbb{P}^{1}$ by the identification $(\tau, z) \sim(\tau+1, z+1)$. A Lipschitz family of spherical metrics on this model is defined by

$$
\|\cdot\|_{\tau}=\frac{\Im \tau|d z|}{|z-\Re \tau|^{2}+\Im^{2} \tau}
$$

It is constructed by starting with the spherical metric $\|\cdot\|_{i}=\frac{|d z|}{1+|z|^{2}}$, which is already invariant by the stabilizer $\operatorname{PSO}(2, \mathbb{R})$ of the point $i$, and then by extending it by the formula $M^{*}\|\cdot\|_{M \tau}=\|\cdot\|_{\tau}$ for any $\tau \in \mathbb{H}$ and $M \in \operatorname{PSL}(2, \mathbb{R})$. The proof of [22, Prop. 2.2] shows that $\|\cdot\|_{\tau}$ is indeed Lipschitz.

The family $\left\{\|\cdot\|_{\tau}\right\}_{\tau \in \mathbb{H}}$ then induces a family of spherical metrics $\left\{\|\cdot\|_{u}\right\}_{u \in \mathbb{D}^{*}}$ on the quotient bundle $\simeq \mathbb{D}^{*} \times \mathbb{P}^{1}$ which is given by the formula

$$
\|\cdot\|_{u}=\frac{\frac{1}{2 \pi} \log \left(\frac{1}{|u|}\right)|d v|}{\left|v+\frac{i}{2 \pi} \log \left(\frac{1}{|u|}\right)\right|^{2}+\frac{1}{4 \pi^{2}} \log ^{2}\left(\frac{1}{|u|}\right)}=\frac{2 \pi}{\log \left(\frac{1}{|u|}\right)} \cdot \frac{|d V|}{\left|\frac{2 \pi}{\log \left(\frac{1}{|u|}\right)}+i V\right|^{2}+|V|^{2}} .
$$

Step 3. The induced singular metric on $N_{\overline{\mathcal{F}}}$.

The family of spherical metrics constructed above on $M_{\sigma}$ induces a metric $\|\cdot\|$ on the normal bundle of the foliation $\overline{\mathcal{F}}$ which possesses singularities along the fibers over the 
cusps of $X$, that we compute here. In the $(u, V)$-coordinates, we have that

$$
\frac{\|\cdot\|}{\|\cdot\|_{s}}=\frac{2 \pi}{|u| \log \left(\frac{1}{|u|}\right)} \cdot \Phi(u, V) \text { where } \Phi(u, V)=\frac{1+|V|^{2}}{\left|\frac{2 \pi}{\log \left(\frac{1}{|u|}\right)}+i V\right|^{2}+|V|^{2}} .
$$

The reader can check that $\Phi$ has a pole only at the point $(u, V)=(0,0)$, extends continuously and extends continuously elsewhere.

Step 4. Defining a foliation index. For any harmonic current $T$ on $\mathbb{D} \times \mathbb{P}^{1}$ directed by $\mathcal{F}_{m}$, we define

$$
I(T):=\int_{\mathbb{D}^{*} \times \mathbb{P}^{1}} \frac{1}{i \pi} \partial \bar{\partial} \Psi \wedge T
$$

where $\Psi: \mathbb{D}^{*} \times \mathbb{P}^{1}$ is a smooth function supported in a domain $D_{r}^{*} \times \mathbb{P}^{1}$ for some $0<r<1$, and such that $\Psi=\log \frac{\|\cdot\|}{\|\cdot\|_{s}}$ in a neighborhood of $0 \times \mathbb{P}^{1}$. Observe that this number does not depend on the chosen function $\Psi$, since the current $T$ is harmonic.

Lemma 3.6. The integral (26) is convergent.

Proof. It suffices to proves the lemma for $\Psi=\log \frac{\|\cdot\|}{\|\cdot\|_{s}}$. In this case the integral $I(T)$ is nothing but the $T$-integral of the differences between the curvature of $\|\cdot\|$ and that of $\|\cdot\|_{s}$. Because $\|\cdot\|_{s}$ is smooth and hence $T$-integrable, it is enough to prove that the curvature of $\|\cdot\|$ is $T$-integrable. We claim that the restriction of the curvature of $\|\cdot\|$ along the leaves is bounded in modulus by the leafwise Poincaré metric. This is sufficient for our purposes since the Poincaré metric is $T$-integrable (due to the fact that $T$ projects on the integration current on $\mathbb{D}$ ) and that the $T$-integral of a $(1,1)$-form depends only on its restriction to $\mathcal{F}$.

To prove this claim, we work in the $(\tau, z)$-uniformizing coordinates, and use formula (23) to get that the curvature of $\|\cdot\|$ along the leaf $\mathbb{H}^{2} \times z$ is

$$
\frac{1}{i} \partial \bar{\partial}_{\tau} \log \left(\frac{\Im \tau}{|z-\Re \tau|^{2}+\Im^{2} \tau}\right) .
$$

Then, writing $\tau=x+i y$, we compute

$$
\frac{1}{i} \partial \bar{\partial}_{\tau} \log \left(\frac{y}{|z-x|^{2}+y^{2}}\right)=\left(\frac{-1}{y^{2}}+\frac{2 \Im^{2} z}{\left((x-\Re z)^{2}+y^{2}+\Im^{2} z\right)^{2}}\right) d x \wedge d y
$$

and the result follows since

$$
0 \leq \frac{2 \Im^{2} z}{\left((x-\Re z)^{2}+y^{2}+\Im^{2} z\right)^{2}} \leq \frac{2}{y^{2}} .
$$

The index is defined so as to have the following formula, which corrects formula (21). For every puncture $p$ of $X$, we define $I(T, p)$ to be the index of the canonical foliated harmonic current defined in subsection 1.5 at the puncture $p$. 
Lemma 3.7. $\chi(\sigma)=\frac{\pi}{\operatorname{vol}(X)}\left(N_{\overline{\mathcal{F}}} \cdot T+\sum_{p} I(T, p)\right)$.

Proof. Let $\Psi$ be the function on $M_{\sigma}$, defined in a neighborhood of the exceptional fibers, as just constructed. Introduce a smooth family of metrics on the fibers of $\bar{M}_{\sigma}$, which coincides with $\|\cdot\|_{s}$ near the punctures. Such a family is not Lipschitz, so we multiply it by a function of the form $e^{\Psi}$, to make it coincide with the local model discussed above, $\|\cdot\|=\|\cdot\|_{s} \cdot e^{\Psi}$. Then we infer that

$$
\int \Theta \wedge T=\int \Theta_{\|\cdot\|_{s}} \wedge T+\int \frac{1}{i \pi} \partial \bar{\partial} \Psi \wedge T=N_{\overline{\mathcal{F}}} \cdot T+\sum_{p} I(T, p)
$$

and result follows from Lemma 3.4.

Step 5. An invariance property for the index

Proposition 3.8. The index $I(T)$ takes the same value on all foliated harmonic currents $T$ on $\mathcal{F}_{m}$ that give mass 1 to the fibers $\{u\} \times \mathbb{P}^{1}$.

Proof. Let us introduce two families of symmetries for the foliation $\mathcal{F}_{m}$. They are induced by the translations $(\tau, z) \mapsto(\tau+x, z)$ and $(\tau, z) \mapsto(\tau, z+c)$ for $x \in \mathbb{R}$ and $c \in \mathbb{C}$ at the level of the universal cover :

$$
H_{x}(u, V)=\left(e^{2 i \pi x} u, \frac{V}{1-x V}\right) \quad \text { and } \quad V_{c}(u, V)=\left(u, \frac{V}{1+c V}\right) .
$$

The following result is the key of the argument:

Lemma 3.9. If $T$ is as in Proposition 3.8, then for all $x \in \mathbb{R}$ and $c \in \mathbb{C}, I\left(\left(H_{x}\right)_{*} T\right)=$ $I\left(\left(V_{c}\right)_{*} T\right)=I(T)$.

Proof. We treat the case of $H_{x}$, the proof being similar (and in fact easier) for $V_{c}$. We have that

$$
I\left(\left(H_{x}\right)_{*} T\right)-I(T)=\int_{\mathbb{D}^{*} \times \mathbb{P}^{1}} \frac{1}{2 i \pi} \partial \bar{\partial}\left(\Psi \circ H_{x}-\Psi\right) \wedge T .
$$

Let us split this function as a sum

$$
\Psi \circ H_{x}-\Psi=\Gamma+\Gamma_{s},
$$

where $\Gamma$ and $\Gamma_{s}$ are smooth functions on $\mathbb{D}^{*} \times \mathbb{P}^{1}$ supported in $\mathbb{D}_{r}^{*} \times \mathbb{P}^{1}$ for some $0<r<1$ and such that in a neighborhood of the divisor $\{u=0\}, \Gamma=\log \frac{\left(H_{x}\right)_{*}\|\cdot\|}{\|\cdot\|}$ and $\Gamma_{s}=\log \frac{\left(H_{s}\right)_{*}\|\cdot\|_{x}}{\|\cdot\|_{s}}$. Observe that

$$
\int \frac{1}{2 i \pi} \partial \bar{\partial} \Gamma_{s} \wedge T=0
$$

since $\|\cdot\|_{s}$ is a smooth metric, thus $\Gamma_{s}$ is a smooth function. Now $\Gamma$ is smooth if $u \neq 0$, tends to 0 uniformly when $u$ tends to 0 , and the derivative of $\Gamma$ along the leaves is bounded 
by the Poincaré metric (since $\|\cdot\|$ is a Lipschitz metric). Since the leafwise Poincaré metric is given in $u$-coordinates by $\frac{|d u|}{|u| \log |u|}$, we get that

$$
\left|d_{\mathcal{F}} \Gamma\right| \leq \frac{|d u|}{|u| \log |u|}
$$

From $(28)$ and $(29)$ we are left to prove that

$$
\int \frac{1}{2 i \pi} \partial \bar{\partial} \Gamma \wedge T=0
$$

(the fact that this integral makes sense follows from Lemma 3.6). To do this, we introduce a family of smooth functions $\theta_{r}: \mathbb{D}^{*} \rightarrow[0,1]$ such that $\theta_{r}(u)=1$ if $|u| \geq r, \theta_{r}(u)=0$ if $|u| \leq r / 2,\left\|d \theta_{r}\right\|_{\infty}=O\left(\frac{1}{r}\right)$, and $\left\|\partial \bar{\partial} \theta_{r}\right\|_{\infty}=O\left(\frac{1}{r^{2}}\right)$. Since $\partial \bar{\partial} \Gamma \wedge T$ is of order 0 , to get (31), it is enough to prove that

$$
\lim _{r \rightarrow 0} \int \theta_{r} \partial \bar{\partial} \Gamma \wedge T=0 .
$$

To compute this integral, we observe that since $T$ is harmonic $\int \partial \bar{\partial}\left(\theta_{r} \Gamma\right) \wedge T=0$, hence we get that

$$
\int \theta_{r} \partial \bar{\partial} \Gamma \wedge T=-\int \Gamma \partial \bar{\partial} \theta_{r} \wedge T-2 \Re \int \partial \theta_{r} \wedge \bar{\partial} \Gamma \wedge T=:-A_{r}-B_{r} .
$$

To conclude the proof, we will show that both integrals $A_{r}$ and $B_{r}$ tend to 0 with $r$. To estimate the former, we write

$$
\left|A_{r}\right| \leq \delta(\Gamma, r) O\left(\frac{1}{r^{2}}\right) \int_{\frac{r}{2} \leq|u| \leq r} i d u \wedge d \bar{u} \wedge T \leq O(\delta(\Gamma, r))
$$

where $\delta(\Gamma, r)=\sup _{\frac{r}{2} \leq|u| \leq r, v \in \mathbb{P}^{1}} \Gamma(u, v)$, and the last inequality holds because $T$ projects on the current of integration on $\mathbb{D}$. As observed above, $\delta(\Gamma, r)=o(1)$ whence $\lim _{r \rightarrow 0} A_{r}=0$. The same argument works for the second integral: indeed by using (30) and the bound on $\left\|d \theta_{r}\right\|_{\infty}$, we get that

$$
\left|B_{r}\right| \leq O\left(\frac{1}{r^{2} \log \left(\frac{1}{r}\right)}\right) \int_{\frac{r}{2} \leq|u| \leq r} i d u \wedge d \bar{u} \wedge T \leq O\left(\frac{1}{\log \left(\frac{1}{r}\right)}\right),
$$

which completes the proof.

Let us resume the proof of Proposition 3.8. Recall from $\$ 1.5$ that a foliated harmonic current $T$ for $\mathcal{F}_{m}$ lifts as a harmonic current $\widetilde{T}$ on $\mathbb{H} \times \mathbb{P}^{1}$, which by the Poisson formula is induced by a family of probability measures $\left\{\nu_{a}\right\}_{a \in \mathbb{R}}$ on $\mathbb{P}^{1}$, depending measurably on $a$, and satisfying the relation $\nu_{a+1}=(z+1)_{*} \nu_{a}$. From this equivariance, the data of such a family of measures is in turn equivalent to that of a probability measure on $[0,1) \times \mathbb{P}^{1}$. Such a measure is a convex combination of Dirac masses on $[0,1) \times \mathbb{P}^{1}$. This shows that any family $\left\{\nu_{a}\right\}$ as above is a convex combination of families of the form $\nu_{\left(a_{0}, z_{0}\right)}, a \in[0,1)$, $z_{0} \in \mathbb{P}^{1}$, where $\left(\nu_{\left(a_{0}, z_{0}\right)}\right)_{a}=0$ if $a \neq a_{0} \bmod$. $\mathbb{Z}$ and $\left(\nu_{\left(a_{0}, z_{0}\right)}\right)_{a_{0}+k}=\delta_{z_{0}+k}$. (Notice that the point $\infty \in \mathbb{P}^{1}$, corresponding to the separatrix of the singularity of $\mathcal{F}_{m}$, plays a special role here. Nevertheless we do not need to take it in to account since our measures and 
currents are diffuse.) Going back to currents, $\nu_{\left(a_{0}, z_{0}\right)}$ corresponds to a certain harmonic current $T_{\left(a_{0}, z_{0}\right)}$ and all foliated harmonic currents for $\mathcal{F}_{m}$ are obtained from these by taking convex combinations.

Now it is clear that $\left(H_{x}\right)_{*}\left(T_{\left(a_{0}, z_{0}\right)}\right)=T_{\left(a_{0}+x, z_{0}\right)}$ and $\left(V_{c}\right)_{*}\left(T_{\left(a_{0}, z_{0}\right)}\right)=T_{\left(a_{0}, z_{0}+c\right)}$, hence we infer from Lemma 3.9 that the index $I$ takes the same value on all the extremal points $T_{\left(a_{0}, z_{0}\right)}$, and we are done.

\section{Proof of Theorem A (and of Proposition 3.3)}

The proof is based on some basic cohomological computations in $H^{2}\left(\bar{M}_{\rho}, \mathbb{C}\right)$. Recall from $\$ 1.1$ that $H^{2}\left(\bar{M}_{\rho}, \mathbb{C}\right)=\mathbb{C}[\bar{s}] \oplus \mathbb{C}[f]$. We will need the following fact: if $\mathcal{G}$ is a singular holomorphic foliation on a complex surface, and $C$ is a non singular compact holomorphic curve that is everywhere transverse to $\mathcal{G}$, then

$$
N_{\mathcal{G}} \cdot C=\mathrm{eu}(C)
$$

Indeed, under these assumptions, $\left.N_{\mathcal{G}}\right|_{C} \simeq T_{C}=-K_{C}$, and by the genus formula, $K_{C} \cdot C=$ $-\mathrm{Eu}(\mathrm{C})$. Hence in our situation, working in $\bar{M}_{\sigma}$ we get that

$$
N_{\overline{\mathcal{F}}} \cdot \bar{s}=\mathrm{eu}(\bar{s})=\mathrm{eu}(\bar{X}) \text { and } N_{\overline{\mathcal{F}}} \cdot f=\mathrm{eu}\left(\mathbb{P}^{1}\right)=2 .
$$

The intersection form in $H^{2}\left(\bar{M}_{\rho}, \mathbb{C}\right)$ is characterized by the identities

$$
\bar{s} \cdot f=1, f^{2}=0 \text {, and } \bar{s}^{2}=\mathrm{eu}(\bar{X}) .
$$

The first two equalities are obvious, and the justification of the third one is as follows: since the section $\bar{s}$ is everywhere tangent to $\mathcal{F}$ and to the fibers, we get an isomorphism between the tangent bundle and normal bundle to $\bar{s}$. Therefore the adjunction formula yields

$$
\bar{s}^{2}=\operatorname{deg}\left(\left.N_{\bar{s}}\right|_{\bar{s}}\right)=-\operatorname{deg}\left(\left.K_{\bar{s}}\right|_{\bar{s}}\right)=\mathrm{eu}(\bar{s})=\mathrm{eu}(\bar{X}) .
$$

From this and $(32)$ we easily deduce that

$$
\left[N_{\overline{\mathcal{F}}}\right]=2[\bar{s}]-\mathrm{eu}(\bar{X})[f] .
$$

Now Proposition 3.5 asserts that

$$
\chi(\sigma)=\frac{1}{2|\mathrm{eu}(X)|}\left(N_{\overline{\mathcal{F}}} \cdot \bar{T}+I \cdot \# P\right) .
$$

Also, from Proposition 2.4 we have that $\delta=\frac{1}{\operatorname{vol}(X)} \bar{T} \cdot \bar{s}$, and it is obvious that $\bar{T} \cdot f=1$. Using the fact that $\mathrm{eu}(X)=\mathrm{eu}(X)+\# P$, altogether this yields

$$
\chi(\sigma)=\frac{1}{2}+2 \pi \delta+(I-1) \frac{\# P}{2|\mathrm{eu}(X)|} .
$$

Therefore, to finish the proof it is enough to show that $I=1$. This is done by considering the particular case of the canonical projective structure induced by the uniformization of $X$, since in this case we have that $\delta=0$ and $\chi=\frac{1}{2}$ (see the remarks following [22, Def. 2.1]). The proof is complete. 


\section{The Dimension of harmonic MeAsure: Proof of TheOrem B}

In this part we provide the proof of Theorem B. We start with a result originating in the work of S. Frankel (see [29]).

Proposition 5.1. Let $\varphi$ be the density of the desintegration of $T$ (resp. $\mu$ ) along the leaves of $\mathcal{F}$ (see (10)). Then the integral

$$
A=-\int_{X_{\rho}} \Delta_{\mathcal{F}} \log \varphi d \mu
$$

is convergent, and moreover

$$
0 \leq A \leq 1
$$

Notice that $\varphi$ is defined only up to a multiplicative factor which is constant along the leaves, which shows that $\Delta_{\mathcal{F}} \log \varphi$ is well defined. The quantity $A$ is called the action of $T$.

Proof. The positivity and harmonicity of the density $\varphi$ implies that $\Delta_{\mathcal{F}} \log \varphi=-\|\nabla \log \varphi\|^{2}$. Thus by the Harnack inequality for positive harmonic functions we infer that $\|\nabla \log \varphi\|$ is uniformly bounded, whence the convergence of the integral in (34). To get the bound (35), we observe that $\varphi$ lifts to the universal cover of $\mu$-a.e. leaf as a positive harmonic function, hence the half-plane version of the Harnack inequality (obtained by taking conjugate harmonic functions and applying the Schwarz-Pick lemma) yields $\|\nabla \log \varphi\| \leq 1$. This proves (35) (see [20] for more details).

The main step of the proof is the following probabilistic estimate of the measure of a ball inside a fiber. For every $x \in M_{\sigma}$, and $\rho>0$, we denote by $B_{\mathbb{P}^{1}}(\tilde{x}, \rho)$ the ball of radius $\rho$ centered at $\tilde{x}$ inside the fiber $\mathbb{P}_{\pi(x)}^{1}$. To ease notation, from now on if $x \in M_{\sigma}$ we denote its fiber by $\mathbb{P}_{x}^{1}$ (resp. the corresponding harmonic measure by $\nu_{x}$ ).

Proposition 5.2. Let $A$ be as in Proposition 5.1. For every $\varepsilon>0$, there exists $r_{\varepsilon}>0$ such that for every $0<r<r_{\varepsilon}$,

$$
\left.\mu\left(x \in M_{\sigma}, \quad \nu_{x}\left(B_{\mathbb{P}^{1}}(x, r)\right) \geq r^{\frac{A}{2 \chi}+\varepsilon}\right)\right) \geq 1-\varepsilon .
$$

Proof. The proof follows an argument of Ledrappier's [47, Thm 4.1, p. 372], with the difference that the discrete random walk is replaced by a cocycle over the ergodic system $\left(\Omega^{\mathcal{F}}, \sigma, W_{\mu}^{\mathcal{F}}\right)$.

In view of the next lemma it is useful to recall that the data of a foliated Brownian path is equivalent to that of a Brownian path in $X$ together with its starting point in the fiber.

Lemma 5.3. Let $x \in X$ and $C_{x}$ be a measurable subset of $\mathbb{P}_{x}^{1}$ such that $\nu_{x}\left(C_{x}\right)>0$. Then for $W_{x}^{X}$-a.e. $\omega:[0, \infty) \rightarrow X$, letting $h_{t}=h\left(\left.\omega\right|_{[0, t]}\right)$, we have that

$$
\limsup _{t \rightarrow \infty}-\frac{\log \nu_{\omega(t)}\left(h_{t}\left(C_{x}\right)\right)}{t} \leq A .
$$


Proof. We first work with foliated Brownian motion. Let us introduce the family of functions $\left(L_{t}\right)_{t \geq 0}$, defined on $\Omega^{\mathcal{F}}$ by

$$
L_{t}(\omega)=-\log \frac{\left(h_{t}\right)^{-1} \nu_{\omega(t)}}{\nu_{\omega}(0)}(\omega(0)) .
$$

It is immediate that $L=\left(L_{t}\right)_{t \geq 0}$ is a cocycle, namely it satifies the relations

$$
L_{t+s}(\omega)=L_{s}(\omega)+L_{t}\left(\sigma_{s}(\omega)\right) \text {, for every } s, t \geq 0 \text { and } \omega \in \Omega^{\mathcal{F}} .
$$

Moreover, in terms of the densities $\varphi, L_{t}$ expresses as $L_{t}(\omega)=-\log \frac{\varphi(\omega(t))}{\varphi(\omega(0))}$, hence by applying the Harnack inequality $\left\|\nabla_{\mathcal{F}} \log \varphi\right\| \leq 1$ we obtain the estimate

$$
\left|L_{t}(\omega)\right| \leq \operatorname{length}\left(\left.\omega\right|_{[0, t]}\right) \text {, for every } t \geq 0 \text { and } \omega \in \Omega^{\mathcal{F}}
$$

(recall that the length in question here is the homotopic length of $\pi(\omega)$ ). Thus the superexponential decay of the heat kernel on the upper half plane [17, §5.7] implies that $L_{t}$ is $W_{\mu}^{\mathcal{F}}$-integrable. The subadditive ergodic theorem applied to the cocycle $L$ and the ergodic system $\left(\Omega^{\mathcal{F}}, \sigma, W_{\mu}^{\mathcal{F}}\right)$ shows that $\frac{L_{t}(\omega)}{t}$ converges a.s. to a limit independent of $\omega$. Arguing exactly as in Lemma 3.4 shows that this limit equals $A$, that is,

$$
\frac{L_{t}(\omega)}{t} \underset{t \rightarrow \infty}{\longrightarrow} A \text {, for } W_{\mu}^{\mathcal{F}} \text {-a.e. } \omega \in \Omega^{\mathcal{F}} \text {. }
$$

From this, we infer that for a.e. $x \in M_{\sigma}$, and $W_{x}^{\mathcal{F}}$ a.e. $\omega$, if $C_{x} \subset \mathbb{P}_{x}^{1}$ is a measurable subset such that $\nu_{x}\left(C_{x}\right)>0$, then

$$
\lim _{t \rightarrow \infty} \frac{1}{\nu_{x}\left(C_{x}\right)} \int_{C_{x}}-\frac{1}{t} \log \left(\frac{\left(h_{t}\right)^{-1} \nu_{\omega(t)}}{\nu_{\omega(0)}}(\omega(0))\right) d \nu_{\omega(0)}=A
$$

This follows from (37), Fubini's theorem and the dominated convergence theorem, since for a generic $\omega$, length $\left(\left.\omega\right|_{[0, t]}\right)=O(t)$ so by (36) the argument of the integral in (38) is bounded independently of $t$. We now use the convexity of the function $-\log$ which by Jensen's inequality implies that

$$
\frac{1}{\nu_{x}\left(C_{x}\right)} \int_{C_{x}}-\frac{1}{t} \log \left(\frac{\left(h_{t}\right)^{-1} \nu_{\omega(t)}}{\nu_{\omega(0)}}(\omega(0))\right) d \nu_{\omega(0)} \geq-\frac{1}{t} \log \left(\frac{\nu_{\omega(t)}\left(h_{t}\left(C_{x}\right)\right)}{\nu_{\omega(0)}\left(C_{x}\right)}\right),
$$

and so we deduce that for a.e. $x \in X$, as soon as $\nu_{x}\left(C_{x}\right)>0$, we have that

$$
\limsup _{t \rightarrow \infty}-\frac{1}{t} \log \nu_{\omega(t)}\left(h_{t}\left(C_{x}\right)\right) \leq A
$$

Notice that this property makes no reference to the starting point in the fiber, so it can be stated as well for a.e. $x \in X$ and $W_{x}^{X}$ a.e. $\omega \in \Omega_{x}^{X}$

To finish the proof it remains to see that this statement holds for every $x$. For this, we first observe that if $C_{x}$ has positive measure, then for $W_{x}^{X}$-a.e. $\omega, \nu_{\omega(1)}\left(h_{1}\left(C_{x}\right)\right)>$ 0 . Furthermore, the distribution of $\omega(1)$ is absolutely continuous, so that the previous estimates hold when $x$ is replaced by $\omega(1)$. The assertion then follows from the Markov property of Brownian motion. 
Let us resume the proof of Proposition 5.2. Fix $\varepsilon>0$, and put $\eta=\frac{4 \chi+A}{4 \chi^{2}} \varepsilon$. By Lemma 3.2 , for $W_{\mu}^{\mathcal{F}}$-a.e. $\omega$, the convergence $\frac{1}{t} \log \left\|D h_{t}(y)\right\| \underset{t \rightarrow \infty}{\longrightarrow} \lambda$, holds uniformly on compact subsets of $\mathbb{P}_{\omega(0)}^{1} \backslash\{r(\omega)\}$. So if we let $R=\frac{1}{2} d_{\mathbb{P}^{1}}(\omega(0), r(\omega))$, there exists $t_{1}=t_{1}(\omega, \varepsilon)$ such that if $t \geq t_{1}$,

$$
h_{t}(B(\omega(0), R)) \subset B\left(\omega(t), e^{(\lambda+\eta) t}\right) .
$$

On the other hand by the previous lemma, for $W_{\mu}^{\mathcal{F}}$ a.e. $\omega$, there exists $t_{2}=t_{2}(\omega, \varepsilon)$ such that for $t \geq t_{2}$

$$
\nu_{\omega(t)}\left(h_{t}(B(\omega(0), \eta)) \geq e^{-(A+\eta) t} .\right.
$$

So we infer that for $t \geq \max \left(t_{1}, t_{2}\right)$,

$$
\nu_{\omega(t)}\left(B\left(\omega(t), e^{(\lambda+\eta) t}\right)\right) \geq e^{-(A+\eta) t} .
$$

For every $t>0$ let $\Omega_{t}$ be the set of paths $\omega \in \Omega$ such that $\max \left(t_{1}, t_{2}\right) \leq t$. Clearly $W_{\mu}^{\mathcal{F}}\left(\Omega_{t}\right) \geq 1-\varepsilon$ for $t \geq t(\varepsilon)$. Setting $r=e^{(\lambda+\eta) t}$, if $\omega \in \Omega_{t}$ and $x=\omega(t)$, for $t \geq t(\varepsilon)$ we have that

$$
\nu_{x}(B(x, r)) \geq r^{-\frac{A+\eta}{\lambda+\eta}}=r^{\frac{A+\eta}{2 \chi-\eta}} .
$$

This finishes the proof since the image of $W_{\mu}^{\mathcal{F}}$ under $\omega \mapsto \omega(t)$ is the measure $\mu$, and $\eta$ was chosen so that $\frac{A+\eta}{2 \chi-\eta}<\frac{A}{2 \chi}+\varepsilon$.

An estimate similar to that of Proposition 5.2 holds in every fiber.

Corollary 5.4. Let $x \in X$ and $\varepsilon>0$. There exists $r_{\varepsilon}>0$ such that if $0<r<r_{\varepsilon}$ then

$$
\nu_{x}\left(\tilde{x} \in \mathbb{P}_{x}^{1}: \nu_{x}\left(B_{\mathbb{P}^{1}}(\tilde{x}, r)\right) \geq r^{\frac{A}{2 \chi}+2 \varepsilon}\right) \geq 1-\varepsilon .
$$

Proof. This is due to the fact that the holonomy map $h_{\gamma}$ corresponding to a path $\gamma$ : $[0,1] \rightarrow X$ of length $\ell$ is bilipschitz with constant depending only on $\ell$, and moreover it sends the measure $\nu_{x}$ to a measure absolutely continuous with respect to $\nu_{y}$, whose density is bounded from above and below by positive constants depending only on $\ell$. Now if $\ell$ is fixed, the proportion of points in a given fiber lying at leafwise distance $\ell$ from a point satisfying the conclusion of Proposition 5.2 tends to 1 when $\varepsilon \rightarrow 0$, so we are done.

We are now ready to finish the proof of Theorem B. Fix a real number $s>\frac{A}{2 \chi}$ and $\varepsilon$ such that $0<2 \varepsilon<s-\frac{A}{2 \chi}$. With $r_{\varepsilon}$ as in Corollary 5.4 , for every $r<r_{\varepsilon}$, consider the set

$$
E_{r, \varepsilon}=\left\{\tilde{x} \in \mathbb{P}_{x}^{1}, \nu_{x}\left(B(\tilde{x}, r) \geq r^{\frac{A}{2 \chi}+2 \varepsilon}\right\} .\right.
$$

From Corollary 5.4 we know that $\nu_{x}\left(E_{r / 5, \varepsilon}\right)>1-\varepsilon$. Furthermore, a classical covering argument gives an estimate of the $s$-dimensional Hausdorff measure of $E_{r / 5, \varepsilon}$. Indeed by the Vitali covering lemma there exists a covering of $E_{r / 5, \varepsilon}$ by balls $B_{\mathbb{P}^{1}}\left(\tilde{x}_{i}, r\right)$ centered on $E_{r / 5, \varepsilon}$ and of radius $r$ such that the corresponding balls of radius $\frac{r}{5}$ are disjoint. This disjointness together with the measure estimate imply that this set of balls has cardinality at most

$$
N \leq\left(\frac{r}{5}\right)^{-\left(\frac{A}{2 \chi}+2 \varepsilon\right)}
$$


Therefore,

$$
\mathcal{H}_{s}\left(E_{r / 5, \varepsilon}\right) \leq \sum_{i}(2 r)^{s} \leq 2^{s} 5^{\frac{A}{2 \chi}+2 \varepsilon} r^{s-\frac{A}{2 \chi}-2 \varepsilon} .
$$

We now set $\varepsilon_{n}=2^{-n}, r_{n}=r\left(\varepsilon_{n}\right) / 5$, and put $F_{k}=\bigcap_{n \geq k} E_{r_{n}, \varepsilon_{n}}$. Since for every $n \geq k$, $F_{k} \subset E_{r_{n}, \varepsilon_{n}}$, from (40) we infer that $\mathcal{H}_{s}\left(F_{k}\right)=0$. On the other hand $\nu_{x}\left(F_{k}\right) \geq 1-\left(\frac{1}{2}\right)^{k-1}$, so if we let $F=\bigcup_{k>1} F_{k}$ we have that $\nu_{x}(F)=1$ and $\mathcal{H}_{s}(F)=0$, hence $\operatorname{dim}_{H}\left(\nu_{x}\right) \leq s$. Since $s>\frac{A}{2 \chi}$ was arbitrary, we conclude that $\operatorname{dim}_{H}\left(\nu_{x}\right) \leq \frac{A}{2 \chi} \leq \frac{1}{2 \chi}$, as asserted.

In particular, it follows from Theorem $\mathrm{A}$ that $\operatorname{dim}_{H}\left(\nu_{x}\right) \leq 1$, and if equality holds then $\delta=0$ and $A=1$. Lemma 5.5 below shows that if $\operatorname{dim}_{H}\left(\nu_{x}\right)=1$, then $\sigma \in \overline{B(X)}$. Conversely, if $\sigma \in \overline{B(X)}$, it follows from Makarov's celebrated theorem [50] that the harmonic measures are supported by a set of dimension 1 (the measures $\nu_{x}$ coincide with the classical harmonic measure in this case, see the next lemma). This completes the proof of the theorem.

Lemma 5.5. Let $\sigma$ be a parabolic projective structure with $\operatorname{deg}(\sigma)=0$. Let $\left(m_{y}\right)_{y \in \operatorname{dev}(\widetilde{\widetilde{X}})}$ be the usual harmonic measure of the open set $\operatorname{dev}(\widetilde{X}) \subset \mathbb{P}^{1}$. Then for every $x \in \widetilde{X}$, $\nu_{x}=m_{\operatorname{dev}(x)}$.

In addition the action $A$ equals 1 if and only if $\operatorname{dev}$ is injective, that is, $\sigma \in \overline{B(X)}$ (see the discussion on the density theorem in 6.1 . .

Proof. The first part is proved by using the conformal invariance of Brownian motion. Indeed, any Brownian path $\eta:\left[0, \infty\left[\right.\right.$ (relative to the spherical metric on $\mathbb{P}^{1}$, say) starting at $y$ hits a.s. the boundary of $\operatorname{dev}(\widetilde{X})$ at a first moment $S>0$. We denote by $p=\eta(S)$. The distribution of $p$ is by definition the harmonic measure $m_{y}$. The path $\left.\eta\right|_{[0, S)}$ can be lifted to a continuous path $\widetilde{\eta}:[0, S) \rightarrow \widetilde{X}$ starting at $\widetilde{\eta}(0)=x$, and satisfying $\operatorname{dev} \circ \widetilde{\eta}=\eta$. Let $\omega:[0, T) \rightarrow \widetilde{X}$ be the reparametrization of $\widetilde{\eta}$ defined by $\omega(t)=\widetilde{\eta}(s)$, where

$$
t=\int_{0}^{s}\left\|D \operatorname{dev}^{-1}(\eta(u))\right\|^{2} d u \text {. }
$$

Here $\mathrm{dev}^{-1}$ is understood as the analytic continuation along $\eta$ of the inverse of dev defined at the neighborhood of $y$ and such that $\operatorname{dev}^{-1}(y)=x$. The conformal invariance can be stated in the following form: $\omega$ is a model for a Brownian path starting at $x$ for the Poincaré metric (see e.g. [14, Section 1]). Since $\omega$ tends to infinity in $\widetilde{X}$ when $t$ tends to $T$, we see that $T=+\infty$ a.s. Moreover, a.s. $\lim _{t \rightarrow+\infty} \operatorname{dev}(\omega(t))=p$, which implies using DefinitionProposition 0.3 that $m_{y}=\nu_{x}$.

Let now address the second part of the lemma. We first prove that $A<1$ if $\operatorname{dev}$ is not injective. We need the concept of an extremal positive harmonic function on the universal covering of $\widetilde{X}$. Such a function is (by definition) the composition of a biholomorphism from $\widetilde{X}$ to $\mathbb{H}$ with the imaginary part function $\Im: \mathbb{H} \rightarrow(0, \infty)$. It will be important to notice that the subgroup of $\operatorname{Aut}(\widetilde{X})$ that preserves an extremal positive function is abelian (this is 
the group of translations in the coordinate where the function is the imaginary part). The following statement is a consequence of the case of equality in the Schwarz-Pick lemma:

A function $\varphi: \widetilde{X} \rightarrow(0, \infty)$ is an extremal positive harmonic function if and only if at some (and hence all) point $x \in \widetilde{X}$ one has $\|\nabla \log \varphi(x)\|=1$.

Now assume that dev is not injective. In such a situation, the covering group $\operatorname{ker}(\mathrm{hol})$ is a non trivial normal subgroup of $\pi_{1}(X)$. Recall (item (ii) of Proposition 1.2) that the family of harmonic measures $\left\{\nu_{x}\right\}_{x \in \widetilde{X}}$ satisfies the equivariance relation $\nu_{\gamma x}=\operatorname{hol}(\gamma)_{*} \nu_{x}$ for every $x \in \tilde{X}$ and every $\gamma \in \pi_{1}(X)$. Hence, the density of the disintegration of $\widetilde{T}$ along the leaves is a function $\varphi: \widetilde{X} \times \mathbb{P}^{1} \rightarrow(0, \infty)$ which belongs to $L_{l o c}^{1}(\mathrm{vol} \otimes \nu)$ and is invariant under the group ker(hol). This subgroup being non trivial and normal, its limit set as a subgroup of isometries of $\operatorname{Aut}(\widetilde{X})$ for the Poincaré metric is the whole $\partial \widetilde{X}$. In particular, it contains non abelian free subgroups [5]. As a consequence, the density $\varphi(\cdot, z)$ of the disintegration of $T$ cannot be extremal. In particular, $\left\|\nabla_{\mathcal{F}} \log \varphi\right\|<1$ a.s. This proves that $A<1$, as required.

It remains to prove that if $\operatorname{dev}$ is injective, then $A=1$. In this case, the holonomy representation is injective with image a discrete subgroup of $\operatorname{PSL}(2, \mathbb{C})$. In particular, using Remark 1.6 we see that the foliated bundle $\left(M_{\sigma}, \mathcal{F}_{\sigma}, T_{\sigma}\right)$ is measurably conjugate to the bundle $\left(M_{\sigma_{\text {Fuchs }}}, \mathcal{F}_{\sigma_{\text {Fuchs }}}, T_{\sigma_{\text {Fuchs }}}\right)$ where $\sigma_{\text {Fuchs }}$ is the uniformizing structure on $X$. Hence $A=A_{\text {Fuchs }}$. But the densities of the disintegration of $T_{\text {Fuchs }}$ along the leaves are given by the Poisson kernel in the uniformization coordinates, in particular these are extremal positive harmonic functions. We conclude that $A=A_{\text {Fuchs }}=1$, and the proof of the lemma is complete.

Remark 5.6. Let $\Omega_{0} \subset \mathbb{P}^{1}$ be any component of the discontinuity set of a finitely generated Kleinian group $\Gamma$. Using Theorem B we can recover the classical Jones-Wolff theorem 41 that the dimension of the harmonic measure of $\partial \Omega_{0}$ is bounded by 1 , with strict inequality unless $\Omega$ is simply connected (see [62] for another dynamical proof of this fact). Indeed, from Lemma 5.5, it is enough to show there exists a Riemann surface $X$ of finite type and a parabolic projective structure on $X$ with zero degree such that $\Omega_{0}=\operatorname{dev}(\widetilde{X})$. This simply follows from the Ahlfors finiteness theorem: first take a finite index torsion free subgroup $\Gamma^{\prime} \subset \Gamma$, and define $X:=\Gamma_{0}^{\prime} \backslash \Omega_{0}$ where $\Gamma_{0}^{\prime} \subset \Gamma^{\prime}$ is the stabilizer of $\Omega_{0}$.

\section{Applications to Teichmüller theory}

6.1. Preliminaries. All this is well-known, but not so easy to locate in the literature when $X$ is non-compact.

Recall that $X$ is assumed to be a Riemann surface of finite type, that is biholomorphic to $\bar{X} \backslash P$ where $\bar{X}$ is compact and $P$ is a finite set of punctures. Introduce a projective structure $\bar{\sigma}$ on $\bar{X}$, which can always can be done e.g. by uniformization.For every projective structure $\sigma$ on $X$, consider the holomorphic quadratic differential on $X$ defined by

$$
q=\{w, x\} d x^{2}
$$

where $x$ and $w$ are projective coordinates for the projective structures $\bar{\sigma}$ and $\sigma$ respectively, and as usual $\{w, x\}$ is the Schwarzian derivative. By the cocycle property of the Schwarzian, 
we infer that $\{w, x\} d x^{2}=\{z, x\} d x^{2}+\{w, z\} d z^{2}$. Remembering that $\{w, x\}$ vanishes if $w$ is a Moebius transformation, we see that the differential $q$ is well-defined, that is does not depend on the chosen coordinates $x$ and $w$. Moreover, a result due to Fuchs and Schwarz shows that a projective structure on $X$ is parabolic if and only if the Laurent series expansion of $q$ at the neighborhood of every point of $P$ takes the form $q(x)=$ $\left(\frac{1}{2 x^{2}}+\right.$ l.o.t. $) d x^{2}$, see [19, Théorème 10.1.1, p. 291]. Hence, the space of parabolic projective structures on $X$ is an complex affine space directed by the set of meromorphic quadratic differentials having poles on $P$ of order at most 1 . This space is the set of sections of the line bundle $L=2 K+O(P)$, where $K$ is the canonical divisor of $\bar{X}$. By Riemann-Roch

$$
h^{0}(\bar{X}, L)-h^{0}(\bar{X}, K-L)=\operatorname{deg}(L)+1-g .
$$

Since $K-L=-K-O(P)$ has no non trivial sections, and that $\operatorname{deg}(L)=4 g-4+|P|$, we deduce

$$
h^{0}(\bar{X}, L)=3 g-3+|P| .
$$

Thus the set of parabolic projective structures on $X$ is a complex affine space of dimension $3 g-3+n$, where $n$ is the number of punctures. Observe that for the once punctured torus or the fourth punctured sphere, the dimension equals 1.

We denote by $T_{g, n}$ the Teichmüller space of equivalence classes of marked Riemann surfaces biholomorphic to a compact Riemann surface of genus $g$ punctured at $n$ distinct points. Here a marking of the Riemann surface $Y$ will refer to the data of a universal covering $\widetilde{Y} \rightarrow Y$ together with an identification of the covering group $\pi_{1}(Y)$ of this covering with $\pi_{1}(X)$. Two marked surfaces are considered as equivalent if there exists an equivariant holomorphic diffeomorphism between the universal covers.

Let $Y \in \mathcal{T}_{g, n}$. Denote by $c(Y)$ the complex conjugation of $Y$, keeping the marking fixed. The Bers simultaneous uniformization theorem [10, Theorem 1] asserts that there exists a faithful discrete representation $\rho_{X, Y}: \pi_{1}(X) \rightarrow \operatorname{PSL}(2, \mathbb{C})$, uniquely defined up to conjugation, such that the Riemann sphere admits a $\rho$-invariant partition of the form

$$
\mathbb{P}^{1}=D_{X} \cup \Lambda \cup D_{Y}
$$

where $D_{X}$ and $D_{Y}$ are two simply connected domains, $\Lambda$ is a topological circle, and such that the marked Riemann surfaces $\rho\left(\pi_{1}(X)\right) \backslash D_{X}$ and $\rho\left(\pi_{1}(X)\right) \backslash D_{Y}$ are respectively equivalent to $X$ and $c(Y)$. A representation with an invariant decomposition such as (42) is called quasi-Fuchsian. Thus an element $Y \in \mathcal{T}_{g, n}$ produces a parabolic $\mathbb{P}^{1}$-structure $b(Y) \in P(X)$ on $X$, defined as the $\rho$-equivariant identification between $\widetilde{X}$ and $D_{X}$.

It turns out that the map $Y \in T_{g, n} \mapsto b(Y) \in P(X)$ is a holomorphic embedding onto a bounded open subset $B(X) \subset P(X)$, known as the Bers embedding (or Bers slice) of $T_{g, n}$. The holomorphicity of $b$ follows from the holomorphic dependence of the solution of the Beltrami equation with respect to parameters. The boundedness of $B(X)$ follows from Nehari's estimate for the Schwarzian of univalent meromorphic functions defined on the hyperbolic disc, and its openness from the so-called Ahlfors-Weill extension lemma (see e.g. [32]). 
Due to deep recent advances in Kleinian group and 3-manifold theory, there is a now good understanding of the structure of $\overline{B(X)}$. To be precise, the density theorem (formerly known as the Bers density conjecture), specialized to our context, asserts that $\sigma \in \bar{B}(X)$ if and only if $\operatorname{dev}_{\sigma}$ is injective. This means in particular that the image of dev is a simply connected component of the discontinuity set of $\operatorname{hol}_{\sigma}\left(\pi_{1}(X)\right)$, which uniformizes $X$. An equivalent formulation is that $\sigma \in \overline{B(X)}$ if and only if $\operatorname{deg}(\sigma)=0$ and hol $\sigma$ is faithful. When $X$ is compact, this was explicitly proved by Bromberg [11. In the general case, this statement is generally accepted by the experts as being a consequence of the ending lamination theorem of Minsky [56] and Brock, Canary and Minsky [9] (see Ohshika [59] and Namazi-Souto [57] for the derivation of the density theorem from the ending lamination theorem in the whole character variety). It seems, however, that no detailed proof of this fact has appeared yet.

6.2. Holomorphic convexity of Bers slices. Here we prove Theorem C. It is known that every component of the interior of a polynomially convex set is polynomially convex (i.e. a Runge domain) (see e.g. [28, Prop. 2.7]), but the converse is false (this fails e.g. for $U=D(0,2) \backslash D(1,1) \subset \mathbb{C})$. In particular the second statement of the theorem (the polynomial convexity of $B(X)$ ) follows from the first (the polynomial convexity of $\overline{B(X)}$ ).

Actually, one may derive the polynomial convexity of $B(X)$ from a simple, direct argument. Indeed, consider in $X$ the set $\{\mathrm{deg}=0\}$ of projective structures with vanishing degree. Equivalently, by Proposition 2.3 such a structure is of quotient type. It was shown in [45] that $\{\operatorname{deg}=0\}$ is a compact subset of $P(X)$. Since in $\mathbb{C}^{n}$ convexity with respect to polynomials and psh functions coincide [66, Thm 1.3.11], we infer that $\{\mathrm{deg}=0\}$ is polynomially convex. Now it is a result due to Shiga and Tanigawa 64] and Matsuzaki [54] that the interior in $P(X)$ of the set of projective structures with discrete holonomy is the set of projective structures with quasifuchsian holonomy. Thus Int $\{\operatorname{deg}=0\}=B(X)$, and the polynomial convexity of $B(X)$ follows.

We now turn to the polynomial convexity of $\overline{B(X)}$. A connected component of a polynomially convex set is polynomially convex [66, Cor. 1.5.5], so it is enough to show that $\overline{B(X)}$ is a connected component of $\{\mathrm{deg}=0\}$. This will be based on the following amusing lemma.

Lemma 6.1. Let $\left(\rho_{\lambda}\right)_{\lambda \in \Lambda}$ be a holomorphic family of representations of a finitely generated group $G$ into $\operatorname{PSL}(2, \mathbb{C})$, parameterized by some complex manifold $\Lambda$. If $K \subset \Lambda$ is a compact connected set of discrete and non-elementary representations then $\operatorname{ker}\left(\rho_{\lambda}\right)$ is constant over $K$. In particular if $K$ contains a faithful representation, then all representations in $K$ are faithful.

Admitting this result for the moment, let us finish the proof. Let $K$ be the connected component of $\{\mathrm{deg}=0\}$ containing $\overline{B(X)}$. By Proposition 2.3 for every $\sigma \in K$, hol $\sigma$ is discrete. Applying Lemma 6.1, we infer that all representations in $K$ are faithful. By the density theorem (see the end of $\$ 6.1$ ), the inclusion $K \subset \overline{B(X)}$ holds, hence $K=\overline{B(X)}$, and the proof is complete. 
Proof of Lemma 6.1. Without loss of generality we may assume that $\Lambda$ is connected. Consider the set of subgroups $\operatorname{ker}\left(\rho_{\lambda}\right)$ as $\lambda$ ranges in $\Lambda$. Our first claim is that this set is at most countable. The argument is based on basic finiteness (Noetherian) properties in analytic geometry. Let $\lambda_{0} \in \Lambda$ and put $K_{0}=\operatorname{ker}\left(\rho_{\lambda_{0}}\right)$. Define

$$
Z^{\prime}\left(K_{0}\right)=\left\{\lambda \in \Lambda, \forall g \in K_{0}, \rho_{\lambda}(g)=\mathrm{id}\right\},
$$

and let $Z\left(K_{0}\right)$ be the component of $Z^{\prime}\left(K_{0}\right)$ containing $\lambda_{0}$. If $\lambda \in Z\left(K_{0}\right), \operatorname{ker}\left(\rho_{\lambda}\right) \supset K_{0}$ nevertheless equality needn't hold. On the other hand we observe that if $\lambda$ is a generic point in $Z\left(K_{0}\right)$, that is, chosen outside a countable family of proper analytic subvarieties, then $\operatorname{ker}\left(\rho_{\lambda}\right)=K_{0}$. Indeed for every $g \in G \backslash K_{0}$, the set $\left\{\lambda \in Z\left(K_{0}\right), \rho_{\lambda}(g)=\right.$ id $\}$ is a proper subvariety of $Z\left(K_{0}\right)$, since it does not contain $\lambda_{0}$.

Conversely, a similar argument shows that if $V \subset \Lambda$ is any irreducible variety, the subgroup

$$
K(V)=\left\{g \in G, \forall \lambda \in V, \rho_{\lambda}(g)=\mathrm{id}\right\}
$$

is the kernel of generic representations in $V$.

So if $K_{0}$ is as above, $Z^{\prime}\left(K_{0}\right)$ has at most countably many irreducible components, each of which associated with a generic kernel (for $Z\left(K_{0}\right)$, this is precisely $K_{0}$ ). Now we observe that locally $Z^{\prime}\left(K_{0}\right)$ is defined by finitely many equations, that is there exists a finite number of elements $g_{i} \in G, i=1 \ldots N$, such that for $\Lambda^{\prime} \Subset \Lambda$,

$$
Z^{\prime}\left(K_{0}\right) \cap \Lambda^{\prime}=\left\{\lambda \in \Lambda^{\prime}, \forall i=1 \ldots N, \rho_{\lambda}\left(g_{i}\right)=\mathrm{id}\right\} .
$$

This leaves only countably many possibilities for the generic kernels, and our claim is proved.

Under the assumptions of the lemma, label all kernels of representations in $K$ as $\left(H_{i}\right)_{i \in \mathbb{N}}$ and write accordingly $K$ as a disjoint union $K=\bigcup K_{i}$, where $K=\left\{\lambda, \operatorname{ker}\left(\rho_{\lambda}\right)=H_{i}\right\}$. The next claim is that for every $i, K_{i}$ is closed. For this we use the precise version of the Chuckrow (Margulis-Zassenhaus-Jorgensen) theorem stated in [42, Thm 8.4 p.170]: if $\rho_{p}$ is a sequence of discrete faithful representations of some non-radical group $\Gamma$ into $\operatorname{PSL}(2, \mathbb{C})$, algebraically converging to some representation $\rho$ of $\Gamma$, then $\rho$ is also discrete and faithful. Recall that a group $\Gamma$ is said non-radical if it does not admit infinite normal nilpotent subgroups. A non-elementary subgroup of PSL $(2, \mathbb{C})$ contains rank 2 non abelian free subgroups and in particular is non-radical.

Let now $\left(\lambda_{p}\right) \in K_{i}^{\mathbb{N}}$ be sequence converging to some $\lambda \in K$. For every $p, \rho_{\lambda_{p}}$ is a discrete faithful non-elementary representation of $G / H_{i}$, which is therefore non-radical. It is clear that $H_{i} \subset \operatorname{ker}\left(\rho_{\lambda}\right)$ so $\rho_{\lambda}$ can be viewed as a representation of $G / H_{i}$. Hence by Chuckrow's theorem $\operatorname{ker}\left(\rho_{\lambda}\right)=H_{i}$, and we conclude that $K_{i}$ is closed.

We have thus written $K$ as an at most countable union of disjoint closed sets $K_{i}$. A (not so well-known!) theorem of Sierpiński [65] asserts that such a decomposition must be trivial. The result is proved.

Remark 6.2. If $\operatorname{dim}(P(X))=1$, it is not necessary to use the density theorem. Indeed in dimension 1, polynomial convexity simply means that $K^{c}$ has no bounded component (there is no such simple topological characterization in higher dimension). What our argument 
says is that $\overline{B(X)}$ is contained in a polynomially convex set $K$ with $\stackrel{\circ}{K}=B(X)$. This directly implies that $\overline{B(X)}^{c}$ has no bounded components (this is left as an exercise to the reader).

\subsection{Exterior powers of the bifurcation current.}

Proof of Theorem $D$. The argument is based on the fact that an isolated minimum of the continuous psh function $\delta$ must belong to $\operatorname{Supp}\left(d d^{c} \delta\right)^{3 g-3}$. This is a consequence of the so-called comparison principle for psh functions [6, Thm A]. A similar idea appears in the work of Bassanelli and Berteloot (see [4, Prop. 6.3]).

It is a theorem due to Hejhal [38, Thm 6] that covering projective structures with Schottky holonomy are isolated points of $\{\delta=0\}$. Therefore, for such a $\sigma_{0}$, we infer that $\sigma_{0} \in \operatorname{Supp}\left(T_{\mathrm{bif}}^{3 g-3}\right)$. Finally, we use a result due to Otal [60] (see also Ito [40]): when $X$ is compact, $\partial B(X)$ is contained in the accumulation set of projective structures with degree 0 and Schottky holonomy. We conclude that $\partial B(X) \subset \operatorname{Supp}\left(T_{\text {bif }}^{3 g-3}\right)$.

Corollary $\mathrm{E}$ is an immediate consequence of the following equidistribution result in the spirit of [22, Thm C]. If $\gamma$ is a closed geodesic on $X$ we let

$$
Z(\gamma, t)=\left\{\sigma \in P(X), \operatorname{tr}^{2}\left(\operatorname{hol}_{\sigma}\right)=t\right\}
$$

(notice that since hol ${ }_{\sigma}$ is well-defined up to conjugacy, so it makes sense to speak of its trace). Fix a sequence $\left(r_{n}\right)_{n \geq 1}$ such that for every $c>0$, the series $\sum e^{-c r_{n}}$ converges. The notion of a random sequence of geodesics of length at most $r_{n}$ was discussed at length in [22]. We say that a holomorphic family of representations $\left(\rho_{\lambda}\right)_{\lambda \in \Lambda}$ is reduced if the associated mapping $\Lambda \rightarrow \mathcal{X}\left(\pi_{1}(X), \operatorname{PSL}(2, \mathbb{C})\right)$ to the character variety has discrete fibers. Notice that since hol $: P(X) \rightarrow \mathcal{X}\left(\pi_{1}(X), \operatorname{PSL}(2, \mathbb{C})\right)$ is injective, this property is satisfied in the context of Corollary $\mathrm{E}$

Proposition 6.3. Let $X$ be a hyperbolic Riemann surface of finite type and $\left(\rho_{\lambda}\right)_{\lambda \in \Lambda}$ be a reduced holomorphic family of non-elementary representations of $\pi_{1}(X)$, with $\operatorname{dim}(\Lambda) \geq k$. Let $\left(r_{n}\right)$ be as above, and fix $t \in \mathbb{C}$. For $i=1, \ldots, k$ fix a sequence $\left(\gamma_{n}^{i}\right)$ of independent random closed geodesics of length at most $r_{n}$. Then almost surely,

$$
\lim _{n_{1} \rightarrow \infty} \cdots \lim _{n_{k} \rightarrow \infty} \frac{1}{4^{k} \prod_{i=1}^{k} \operatorname{length}\left(\gamma_{n_{i}}^{i}\right)}\left[Z\left(\gamma_{n_{1}}^{1}, t\right) \cap \cdots \cap Z\left(\gamma_{n_{k}}^{k}, t\right)\right]=T_{\text {bif }}^{k},
$$

Note that in 43 the intersections are counted with multiplicity (i.e. in the sense of holomorphic chains, see [15, Chap. 12]).

Proof. The proof is similar to that of [25, Thm 6.16]. We argue by induction on $k$. For $k=1$ this is [22, Thm C]. Now assume that the result has been proved for $k$. Since $T_{\text {bif }}$ has continuous potential, it follows from 43 that

$$
\frac{1}{4^{k} \prod_{i=1}^{k} \operatorname{length}\left(\gamma_{n_{i}}^{i}\right)}\left[Z\left(\gamma_{n_{1}}^{1}, t\right) \cap \cdots \cap Z\left(\gamma_{n_{k}}^{k}, t\right)\right] \wedge T_{\text {bif }}
$$


converges to $T_{\mathrm{bif}}^{k+1}$ as $n_{k}, \ldots, n_{1} \rightarrow \infty$ successively. Now, when $n_{1}, \ldots, n_{k}$ are large and fixed, we apply [22, Thm C] to the family $\left(\rho_{\lambda}\right)_{\lambda \in Z\left(\gamma_{n_{1}}^{1}, t\right) \cap \cdots \cap Z\left(\gamma_{n_{k}}^{k}, t\right)}$ (the reducedness assumption is used here to ensure that this family is non constant) to get that

$$
\begin{aligned}
\lim _{n_{k+1} \rightarrow \infty} \frac{1}{4^{k+1} \prod_{i=1}^{k+1} \operatorname{length}\left(\gamma_{n_{i}}^{i}\right)} & {\left[Z\left(\gamma_{n_{1}}^{1}, t\right) \cap \cdots \cap Z\left(\gamma_{n_{k+1}}^{k+1}, t\right)\right] } \\
& =\frac{1}{4^{k} \prod_{i=1}^{k} \operatorname{length}\left(\gamma_{n_{i}}^{i}\right)}\left[Z\left(\gamma_{n_{1}}^{1}, t\right) \cap \cdots \cap Z\left(\gamma_{n_{k}}^{k}, t\right)\right] \wedge T_{\text {bif }}
\end{aligned}
$$

and we are done.

\section{Appendix A. Branched projective stuctures}

A branched $\mathbb{P}^{1}$-structure on $X$ is by definition an equivalence class of developmentholonomy pairs (dev, hol), where hol is a representation of $\pi_{1}(X)$ with values in $\operatorname{PSL}(2, \mathbb{C})$ and dev $: \widetilde{X} \rightarrow \mathbb{P}^{1}$ is a hol-equivariant (non constant) meromorphic map. Two developmentholonomy pairs are considered as equivalent if they are of the form $(\mathrm{dev}, \mathrm{hol})$ and $(A \circ$ $\operatorname{dev}, A \circ$ hol $\left.\circ A^{-1}\right)$ for some $A \in \operatorname{PSL}(2, \mathbb{C})$. Thus the only difference with the classical (unbranched) case is that the developing maps are allowed to have critical points. These points are organized as a finite number of orbits under $\pi_{1}(X)$, and their projections in $X$ are called the branched points. If $X$ is a punctured Riemann surface, we can define a notion of branched parabolic $\mathbb{P}^{1}$-structure exactly as before, by specifying it to be induced by $\log z$ near the cusps.

Examples of branched $\mathbb{P}^{1}$-structures come from conformal metrics with constant curvature $-1,0,1$ on $X$ and conical angles multiple of $2 \pi$. In particular, a non constant meromorphic map from $X$ to $\mathbb{P}^{1}$ defines a branched $\mathbb{P}^{1}$-structure with trivial holonomy. Quadratic differentials are other kind of examples associated with a flat metric. We refer more generally to [68]. Those examples of non negative curvature have elementary holonomies. Nevertheless, the holonomy of a branched $\mathbb{P}^{1}$-structure induced by a conformal metric of curvature -1 and conical angle multiple of $2 \pi$ is always non elementary. We refer to [67] for the construction of many examples.

When $X$ is compact, Gallo, Kapovich and Marden [31] showed that if $\rho: \pi_{1}(X) \rightarrow$ $\operatorname{PSL}(2, \mathbb{C})$ is a non elementary representation which does not lift to $\operatorname{SL}(2, \mathbb{C})$, then $\rho$ is the holonomy of a branched $\mathbb{P}^{1}$ - structure with exactly one branch point of angle $4 \pi$ (for a certain Riemann surface structure on $X$ which depends on $\rho$ ). On the other hand $\rho$ is not the holonomy of a unbranched $\mathbb{P}^{1}$-structure.

Some of our results extend mutatis mutandis to branched projective structures with non elementary holonomy. For instance, the degree of a branched $\mathbb{P}^{1}$-structure is defined exactly as in Definition-Proposition 0.1, the proof being identical to the unbranched case. The Lyapunov exponent depends only on the Riemann surface structure and on the holonomy representation, hence it has already been defined in our previous work [22]. Finally, Hussenot's definition of the harmonic measures was actually introduced in the context of branched $\mathbb{P}^{1}$-structures with non elementary holonomy. 
In this appendix we indicate how our formula relating the Lyapunov exponent to the degree needs to be modified in the branched case.

Theorem F. Let $\sigma$ be a parabolic branched $\mathbb{P}^{1}$ structure on a hyperbolic Riemann surface $X$ of finite type. Let $k$ denote the number of branched points, counted with multiplicity. Then, with notation as in Theorem $A$, the following formula holds:

$$
\chi(\sigma)=\frac{1}{2}+2 \pi \delta(\sigma)-\frac{k}{|\mathrm{eu}(\mathrm{X})|}=\frac{1}{2}+\frac{\operatorname{deg}(\sigma)-k}{|\mathrm{eu}(\mathrm{X})|}
$$

Sketch of proof. Introduce as in the unbranched case the flat bundle $\left(\overline{M_{\sigma}}, \overline{\mathcal{F}_{\sigma}}\right)$, the holomorphic section $\bar{s}$ (the compactification of the graph of the developing map at the level of the universal cover) and the normalized harmonic current $\bar{T}$ giving mass 1 to the generic fibers. Proposition 3.5 holds without modification, as well as the computation of the index $I$ made in $\$ 4$ (which is local near the punctures), so we infer that

$$
\chi(\sigma)=\frac{1}{2|\mathrm{eu}(X)|}\left(N_{\overline{\mathcal{F}}} \cdot \bar{T}+\# P\right) .
$$

Now if $\mathcal{G}$ is a singular holomorphic foliation on a complex surface, and $C$ is a non singular compact holomorphic curve not everywhere tangent to $\mathcal{G}$, and not intersecting the singular set of $\mathcal{G}$, we have

$$
N_{\mathcal{G}} \cdot C=\mathrm{eu}(C)+|\operatorname{tang}(\mathcal{G}, C)|,
$$

where the tangency points are counted with multiplicities (see 12 for details). Hence formula (32) has to be replaced by

$$
N_{\overline{\mathcal{F}}} \cdot \bar{s}=\mathrm{eu}(\bar{s})+|\operatorname{tang}(\overline{\mathcal{F}}, \bar{s})|=\mathrm{eu}(\bar{X})+k \text { and } N_{\overline{\mathcal{F}}} \cdot f=\mathrm{eu}\left(\mathbb{P}^{1}\right)=2 .
$$

We also have

$$
\bar{s}^{2}=\mathrm{eu}(\bar{s})+|\operatorname{tang}(\overline{\mathcal{F}}, \bar{s})|=\mathrm{eu}(\bar{s})+k .
$$

So we infer that

$$
\left[N_{\overline{\mathcal{F}}}\right]=2[\bar{s}]-(\mathrm{eu}(\bar{X})+k)[f],
$$

and we conclude as in the proof of Theorem $\mathrm{A}$.

\section{REFERENCES}

[1] Ahlfors, Lars, Finitely generated Kleinian groups, American Journal of Mathematics 86 (1964) 413-429

[2] Alvarez, Sébastien, Discretization of harmonic measures for foliated bundles. C. R. Math. Acad. Sci. Paris 350 (2012), no. 11-12, 621-626.

[3] Barth, W.; Peters, C.; Van de Ven, A. Compact complex surfaces. Ergebnisse der Mathematik und ihrer Grenzgebiete (3), 4. Springer-Verlag, Berlin, 1984. x+304 pp.

[4] Bassanelli, Giovanni; Berteloot, François. Bifurcation currents in holomorphic dynamics on $\mathbf{P}^{k}$. J. Reine Angew. Math. 608 (2007), 201-235.

[5] Beardon, Alan F. The geometry of discrete groups. Graduate Texts in Mathematics, 91. SpringerVerlag, New York, 1983.

[6] Bedford, Eric; Taylor, B. Alan. The Dirichlet problem for a complex Monge-Ampère equation. Invent. Math. 37 (1976), 1-44. 
[7] Berndtsson, Bo; Sibony, Nessim. The $\bar{\partial}$-equation on a positive current. Invent. Math. 147 (2002), no. 2, 371-428.

[8] Bonatti, Christian; Gómez-Mont, Xavier. Sur le comportement statistique des feuilles de certains feuilletages holomorphes. Monographie de l'Enseignement Mathématique. 38 (2001) 15-41.

[9] Brock, Jeffrey F.; Canary, Richard D.; Minsky, Yair N. The classification of Kleinian surface groups, II: The Ending Lamination Conjecture, Ann. Math. 176 (2012), 1-149.

[10] Bers, Lipman. Simultaneous uniformization. Bull. Amer. Math. Soc. 661960 94-97.

[11] Bromberg, Kenneth. Projective structures with degenerate holonomy and the Bers density conjecture. Ann. Math. 166 (2007), 77-93.

[12] Brunella, Marco. Birational geometry of foliations. Publ. Mat. do IMPA, Rio de Janeiro, 2004.

[13] Candel, Alberto. The harmonic measures of Lucy Garnett. Adv. Math. 176 (2003), no. 2, $187-247$.

[14] Carne, T. K. Brownian motion and Nevanlinna theory. Proc. London Math. Soc. (3) 52 (1986), 349-368.

[15] Chirka, Evgueny M. Complex analytic sets. Mathematics and its Applications (Soviet Series), 46. Kluwer Academic Publishers Group, Dordrecht, 1989.

[16] Calsamiglia, Gabriel; Deroin, Bertrand; Frankel, Sidney; Guillot, Adolfo. Singular sets of holonomy maps for singular foliations. J. Eur. Math. Soc 15 (2013), 1067-1099.

[17] Davies, Edward B. Heat kernels and spectral theory. Cambridge Tracts in Mathematics, 92. Cambridge University Press, Cambridge, 1989.

[18] Dabbek, Khalifa; Elkhadhra, Fredj; El Mir, Hassine. Extension of plurisubharmonic currents. Math. Z. 245 (2003), no. 3, 455-481.

[19] de St Gervais, Henri Paul. Uniformisation des surfaces de Riemann : retour sur un théorème centenaire. ENS Éditions, Lyon, 2010.

[20] Deroin, Bertrand. Hypersurfaces Levi-plates immergées dans les surfaces complexes de courbure positive. Ann. Sci. École Norm. Sup. (4) 38 (2005), no. 1, 57-75.

[21] Deroin, Bertrand; Dujardin, Romain. Random walks, Kleinian groups, and bifurcation currents. Invent. Math. 190 (2012), no. 1, 57-118.

[22] Deroin, Bertrand; Dujardin, Romain. Lyapunov exponents for surface group representations. Preprint (2013). math.GT: 1305.0049

[23] Deroin, Bertrand; Dupont, Christophe. Topology and dynamics of Levi-flats in surfaces of general type. Preprint (2012). math. CV:1203.6244

[24] Deroin, Bertrand; Kleptsyn, Victor. Random conformal dynamical systems. Geom. Funct. Anal. 17 (2007), no. 4, 1043-1105.

[25] Dujardin, Romain; Favre, Charles. Distribution of rational maps with a preperiodic critical point. Amer. J. Math. 130 (2008), 979-1032.

[26] Dujardin, Romain. Bifurcation currents and equidistribution in parameter space. Preprint (2011), to appear in Frontiers in complex dynamics (celebrating John Milnor's 80th birthday).

[27] Dumas, David. Complex projective structures. In Handbook of Teichmüller Theory, Volume II. Ed. Athanase Papadopoulos. EMS, 2009.

[28] Fornæss, John Erik; Sibony, Nessim. Complex Hénon mappings in $\mathbb{C}^{2}$ and Fatou-Bieberbach domains. Duke Math. J. 65 (1992), 345-380.

[29] Frankel Sidney. Harmonic analysis of surface group representations in Diff( $\left.\mathbb{S}^{1}\right)$ and Milnor type inequalities. Prépub. 1125 de l'École Polytechnique. 1996.

[30] Furstenberg, Harry Boundary theory and stochastic processes on homogeneous spaces. Harmonic analysis on homogeneous spaces (Proc. Sympos. Pure Math., Vol. XXVI, Williams Coll., Williamstown, Mass., 1972), 193-229. Amer. Math. Soc., Providence, R.I., 1973.

[31] Gallo, Daniel; Kapovich, Michael; Marden, Albert The monodromy groups of Schwarzian equations on closed Riemann surfaces. Ann. of Math. (2) 151 (2000), no. 2, 625-704. 
[32] Gardiner, Frederick P.; Lakic, Nikola. Quasiconformal Teichmüller theory. Mathematical Surveys and Monographs, 76. American Mathematical Society, Providence, RI, 2000.

[33] Garnett, Lucy. Foliations, the ergodic theorem and Brownian motion. J. Funct. Anal. 51 (1983), no. 3, 285-311.

[34] Ghys, Étienne. Laminations par surfaces de Riemann. Dynamique et géométrie complexes (Lyon, 1997), ix, xi, 49-95, Panor. Synthèses, 8, Soc. Math. France, Paris, 1999.

[35] Goldman, William. Projective structures with Fuchsian holonomy. J. Diff. Geom. 25 (3) 297-326, 1987.

[36] Gunning, Robert C. Special coordinate coverings of Riemann surfaces. Math. Ann. $170196767-86$.

[37] Hejhal, Dennis A. Monodromy groups and linearly polymorphic functions. Acta Math. 135 (1975), no. $1,1-55$.

[38] Hejhal, Dennis A. On Schottky and Koebe-like uniformizations. Duke Math. J. 55 (1987), 267-286.

[39] Hussenot, Nicolas. Analytic continuation of holonomy germs of Riccati foliations along brownian paths. Preprint (2013).

[40] Ito, Kentaro. Schottky groups and Bers boundary of Teichmüller space. Osaka J. Math. 40 (2003), no. 3, 639-657.

[41] Jones, Peter W.; Wolff, Thomas. H. Hausdorff dimension of harmonic measures in the plane. Acta Math. 161 (1988), no. 1-2, 131-144.

[42] Kapovich, Michael. Hyperbolic manifolds and discrete groups. Progress in Mathematics, 183. Birkhauser Boston, Inc., Boston, MA, 2001.

[43] Kra, Irwin. Deformations of Fuchsian groups. Duke Math. J. 361969 537-546.

[44] Kra, Irwin. Deformations of Fuchsian groups. II. Duke Math. J. 381971 499-508.

[45] Kra, Irwin; Maskit, Bernard. Remarks on projective structures. Riemann surfaces and related topics: Proceedings of the 1978 Stony Brook Conference, pp. 343-359, Ann. of Math. Stud., 97, Princeton Univ. Press, Princeton, N.J., 1981.

[46] Lecuire, Cyril. Modèles et laminations terminales (d'après Minsky et Brock-Canary-Minsky). Séminaire N. Bourbaki 1068 (mars 2013).

[47] Ledrappier, François. Quelques propriétés des exposants caractéristiques. École d'été de probabilités de Saint-Flour, XII-1982, 305-396, Lecture Notes in Math., 1097, Springer, Berlin, 1984.

[48] Ledrappier, François Une relation entre entropie, dimension et exposant pour certaines marches aléatoires. C. R. Acad. Sci. Paris Sér. I Math. 296 (1983), no. 8, 369-372.

[49] Loray, Frank; Marín Pérez, David. Projective structures and projective bundles over compact Riemann surfaces. Astérisque No. 323 (2009), 223-252.

[50] Makarov, Nikolai G. Distortion of boundary sets under conformal mappings. Proc. London Math. Soc. (3) 51 (1985), 369-384.

[51] Manning, Anthony. The dimension of the maximal measure for a polynomial map. Ann. of Math. (2) 119 (1984), no. 2, 425-430.

[52] Margulis, Grigoriy A. On some aspects of the theory of Anosov systems. With a survey by Richard Sharp. Springer Monographs in Mathematics. Springer-Verlag, Berlin, 2004.

[53] Margulis, Grigorii A. Arithmeticity of the irreducible lattices in the semisimple groups of rank greater than 1. Invent. Math. 76 (1984), no. 1, 93-120.

[54] Matsuzaki, Katsuhiko. The interior of discrete projective structures in the Bers fiber. Ann. Acad. Sci. Fenn. Math. 32 (2007), 3-12.

[55] McMullen, Curtis T. Renormalization and 3-manifolds which fiber over the circle. Annals of Mathematics Studies, 142. Princeton University Press, Princeton, NJ, 1996.

[56] Minsky, Yair N. The classification of punctured-torus groups. Ann. of Math. (2) 149 (1999), 559626.

[57] Namazi, Hossein; Souto, Juan. Non-realizability and ending laminations: proof of the density conjecture. Acta Math. 209 (2012), 323-395. 
[58] Nevalinna, Rolf. Analytic functions. Grundlehren der Mathematischen Wissenschaften, 162, Springer-Verlag, New York-Berlin, 1970.

[59] Ohshika, Ken'ichi. Realising end invariants by limits of minimally parabolic, geometrically finite groups. Geom. Topol. 15 (2011), 827-890.

[60] Otal, Jean-Pierre. Sur le bord du prolongement de Bers de l'espace de Teichmüller. C. R. Acad. Sci. Paris Sér. I Math. 316 (1993), 157-160.

[61] Przytycki, Feliks. Hausdorff dimension of harmonic measure on the boundary of an attractive basin for a holomorphic map. Invent. Math. 80 (1985), no. 1, 161-179.

[62] Przytycki, Feliks; Urbanski, Mariusz; Zdunik, Anna. Harmonic, Gibbs and Hausdorff measures on repellers for holomorphic maps. I. Ann. of Math. (2) 130 (1989), no. 1, 1-40.

[63] Shiga, Hiroshige. On analytic and geometric properties of Teichmüller spaces. J. Math. Kyoto Univ. 24 (1984), no. 3, 441-452.

[64] Shiga, Hiroshige; Tanigawa, Harumi. Projective structures with discrete holonomy representations. Trans. Amer. Math. Soc. 351 (1999), no. 2, 813-823.

[65] Sierpiński, Wacław. Un théorème sur les continus. Tohoku Math. J. 13 (1918), 300-305.

[66] Stout, Edgar Lee. Polynomial convexity. Progress in Mathematics, 261. Birkhäuser Boston, Inc., Boston, MA, 2007.

[67] Troyanov, Marc. Prescribing curvature on compact surfaces with conical singularities. Trans. AMS 324 (1991) no. 2, 793-821.

[68] Veech, William A. Flat surfaces. Amer. J. Math. 115 (1993), no. 3, 589-689.

CNRS, Département de Mathématique d'Orsay, BÂtiment 425, Université de Paris Sud, 91405 ORSAY CEDEX, FRANCE.

E-mail address: bertrand.deroin@math.u-psud.fr

LAMA, Université Paris-Est Marne-la-Vallée, 5 Boulevard Descartes, 77454 Champs SUR MARNE, FrancE

E-mail address: romain.dujardin@univ-mlv.fr 